\title{
A novel PKC activating molecule promotes neuroblast differentiation and delivery of newborn neurons in brain injuries
}

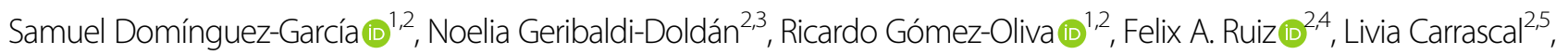
Jorge Bolívar 2,6, Cristina Verástegui, ${ }^{2,3}$, Monica Garcia-Alloza 1,2, Antonio J. Macías-Sánchez 2,7, Rosario Hernández-Galán 2,7, Pedro Nunez-Abades ${ }^{2,5}$ and Carmen Castro (1) ${ }^{1,2}$

\begin{abstract}
Neural stem cells are activated within neurogenic niches in response to brain injuries. This results in the production of neuroblasts, which unsuccessfully attempt to migrate toward the damaged tissue. Injuries constitute a gliogenic/nonneurogenic niche generated by the presence of anti-neurogenic signals, which impair neuronal differentiation and migration. Kinases of the protein kinase C (PKC) family mediate the release of growth factors that participate in different steps of the neurogenic process, particularly, novel PKC isozymes facilitate the release of the neurogenic growth factor neuregulin. We have demonstrated herein that a plant derived diterpene, (EOF2; CAS number 223080606-9), with the capacity to activate PKC facilitates the release of neuregulin 1, and promotes neuroblasts differentiation and survival in cultures of subventricular zone (SVZ) isolated cells in a novel PKC dependent manner. Local infusion of this compound in mechanical cortical injuries induces neuroblast enrichment within the perilesional area, and noninvasive intranasal administration of EOF2 promotes migration of neuroblasts from the SVZ towards the injury, allowing their survival and differentiation into mature neurons, being some of them cholinergic and GABAergic. Our results elucidate the mechanism of EOF2 promoting neurogenesis in injuries and highlight the role of novel PKC isozymes as targets in brain injury regeneration.
\end{abstract}

\section{Introduction}

Brain injuries of different etiologies, produce sensorymotor alterations and irreversible cognitive deficits ${ }^{1}$. No effective treatments to compensate neuronal loss have been found so far. However, the discovery of neurogenesis in the adult brain revealing the capacity of the brain to generate new neurons from neural stem cells (NSC) has opened a door to new therapies to effectively treat

\footnotetext{
Correspondence: Pedro Nunez-Abades (pnunez@us.es) or

Carmen Castro (carmen.castro@uca.es)

'Área de Fisiología, Facultad de Medicina, Universidad de Cádiz, Cádiz, Spain ${ }^{2}$ Instituto de Investigación e Innovación Biomédica de Cádiz (INiBICA), Cádiz, Spain

Full list of author information is available at the end of the article.

These authors contributed equally: Samuel Domínguez-García, Noelia Geribaldi-Doldán

Edited by M. Agostini
}

this type of disorders. Adult neurogenesis occurs under physiological conditions in the dentate gyrus of the hippocampus (DG) and the subventricular zone $(\mathrm{SVZ})^{2,3}$. These regions also react to an injury activating NSC to produce neural progenitor cells (NPC), stimulating proliferation and differentiation of NPC into neuroblasts, and altering migration patterns of these neuroblasts to lead them towards the injured area ${ }^{4-6}$. However, only a very small number of newly born neurons reach the lesion $^{7-9}$. This is a consequence of the release of inflammatory signals that create a gliogenic/non-neurogenic environment within the injured tissue ${ }^{10}$. For example, ligands of the epidermal growth factor receptor (EGFR) in injuries promote gliogenesis and impair migration of neuroblasts towards injuries ${ }^{11}$. On the contrary, inhibition of EGFR ligand release facilitates 
neurogenesis in injuries ${ }^{12-14}$. Transforming growth factor alpha (TGF $\alpha$ ), and other EGFR ligands are synthesized as precursor membrane anchored proteins, which are secreted upon the metalloprotease-catalyzed proteolysis of the soluble, active ectodomain. ADAM17 is the main convertase involved in the ectodomain shedding of TGF $\alpha$ and other EGFR ligands ${ }^{15-17}$. However, ADAM17 also catalyzes the proteolysis of ligands, that activate other receptors of the ErbB family such as neuregulins. The selectivity of this enzyme for each ligand governs ligand release and it is determined by phosphorylation reactions within the cytoplasmic domain of the proligand molecules, catalyzed by kinases of the protein kinase C (PKC) family ${ }^{18}$. PKC $\alpha$ activated by phorbol-12myristate-13-acetate (PMA) catalyzes the phosphorylation of TGF $\alpha$, amphiregulin and HB-EGF precursors facilitating their ADAM17-mediated shedding. On the contrary, activation of novel $\mathrm{PKC} \delta$ is required for ADAM17-mediated secretion of neuregulin 1 $(\mathrm{NRG1})^{18,19}$. Neuregulin-mediated activation of ErbB4 favors neurogenesis in the adult brain promoting neuroblast survival and organizing migration of neuroblasts from the SVZ towards the olfactory bulb ${ }^{20,21}$. Considering these findings, it seems reasonable to hypothesize that specific activation of different PKC isozymes would alter the pattern of growth factor secretion within neurogenic niches, and will regulate either proliferation-mediated by classical PKC $\beta$ and $\alpha^{22}$-or neuroblast survival and migration-mediated by novel PKC isozymes ${ }^{23}$. Consequently, in order to promote neurogenesis in injuries, it would be useful to find molecules with the capacity to activate novel PKC specifically facilitating neuregulins release. Such molecules would probably promote differentiation of NPC into newborn neurons and enable neuroblasts migration from neurogenic areas towards injuries.

\section{Results}

The diterpene with lathyrane structure EOF2 activates PKC without promoting proliferation

The lathyrane EOF2 (CAS number 2230806-06-9) was previously described by our group as a diterpene with no effect on NPC proliferation ${ }^{22}$. Since other diterpenes induced proliferation of $\mathrm{NPC}^{24}$ via classical PKC activation $^{23}$, we analyzed its capacity to activate PKC and to facilitate the generation of neurospheres. Although EOF2 treatment increased PKC activity by twofold in neurosphere cultures (supplementary fig. S1 D), it exerted no change in the size of the neurospheres and a reduction in the number of neurospheres generated. Given its capacity to activate PKC without inducing proliferation, it was possible that EOF2 specifically activated novel PKC without affecting the classical isozymes-reported as responsible of the proliferative effect ${ }^{22}$.

\section{EOF2 facilitates NRG1 release}

In order to determine whether EOF2 specifically induced neuregulin release, we used protein constructs in which NRG1 was fused to mCherry in the N-terminal and eGFP in the C-terminal (mCherry-NRG1-eGFP) (Fig. 1A) according to the work of Kamezaki et al. with modifications $^{25}$. A similar construct was used to test TGF $\alpha$ release (mCherry-TGF $\alpha$-eGFP) (Fig. 2A). We tested the effect of EOF2 in HEK293T cells transfected with the constructs. As shown in supplementary table T1, HEK293T cells express the mRNAs encoding for PKC isozymes involved in neuregulins and TGF $\alpha$ release as well as ADAM17 protein, validating them as a model to test the release of the above-mentioned growth factors.

NRG1 release was measured as explained in M\&M section. As shown in Fig. 1 the red/green ratio in HEK293T transfected with mCherry-NRG1-eGFP and treated with vehicle (none) did not vary with time (Fig. 1B, C) (supplementary movie 1), indicating that in the absence of a PKC-stimulating compound this ligand was not released. However, the addition of EOF2 induced a progressive reduction of this ratio (Fig. 1B, C) (supplementary movie 2), suggesting that this compound was facilitating the mCherry-labeled ectodomain shedding of neuregulin. Interestingly, these changes in color ratio were prevented by addition of the general PKC inhibitor Gö6850 to the culture medium (at a final concentration of $1 \mu \mathrm{M}), 30 \mathrm{~min}$ before the beginning of the treatment with EOF2 (Fig. 1B, C) (supplementary movie 3). In order to uncertain the role that novel PKC played in this effect, we added the classical PKC inhibitor Gö6976 (at a final concentration of $1 \mu \mathrm{M}), 30 \mathrm{~min}$ before the addition of EOF2 to mainly inhibit classical PKC. Results show a reduction in the red/green ratio even in the presence of the inhibitor, indicating that the effect on neuregulin release was mediated by novel PKC activation (Fig. 1A-C) (supplementary movie 4).

Furthermore, we analyzed the levels of mCherry signal in the culture medium of HEK293T cells transfected with the mCherry-NRG1-eGFP construct and treated with EOF2, vehicle, or EOF2 + Gö6850. 30, 60, and $180 \mathrm{~min}$ after the treatment cells were separated from the culture medium and mCherry fluorescence was measured in a fraction of $50 \mu \mathrm{L}$ of medium (total volume of medium $1 \mathrm{~mL}$ ) as a ratio of the GFP fluorescence remaining inside the cells. The red/green ratio in control cultures (non-treated transfected cells) at all time points was considered as $100 \%$ (Fig. 1D dashed line). A time-dependent increase in the red/green fluorescence ratio was observed in the culture medium upon treatment with EOF2. This was not observed in cultures treated with EOF2 + Gö6850 (Fig. 1D).

In order to analyze whether EOF2 specifically facilitated the shedding of NRG1, and had no effect on TGF $\alpha$, we 


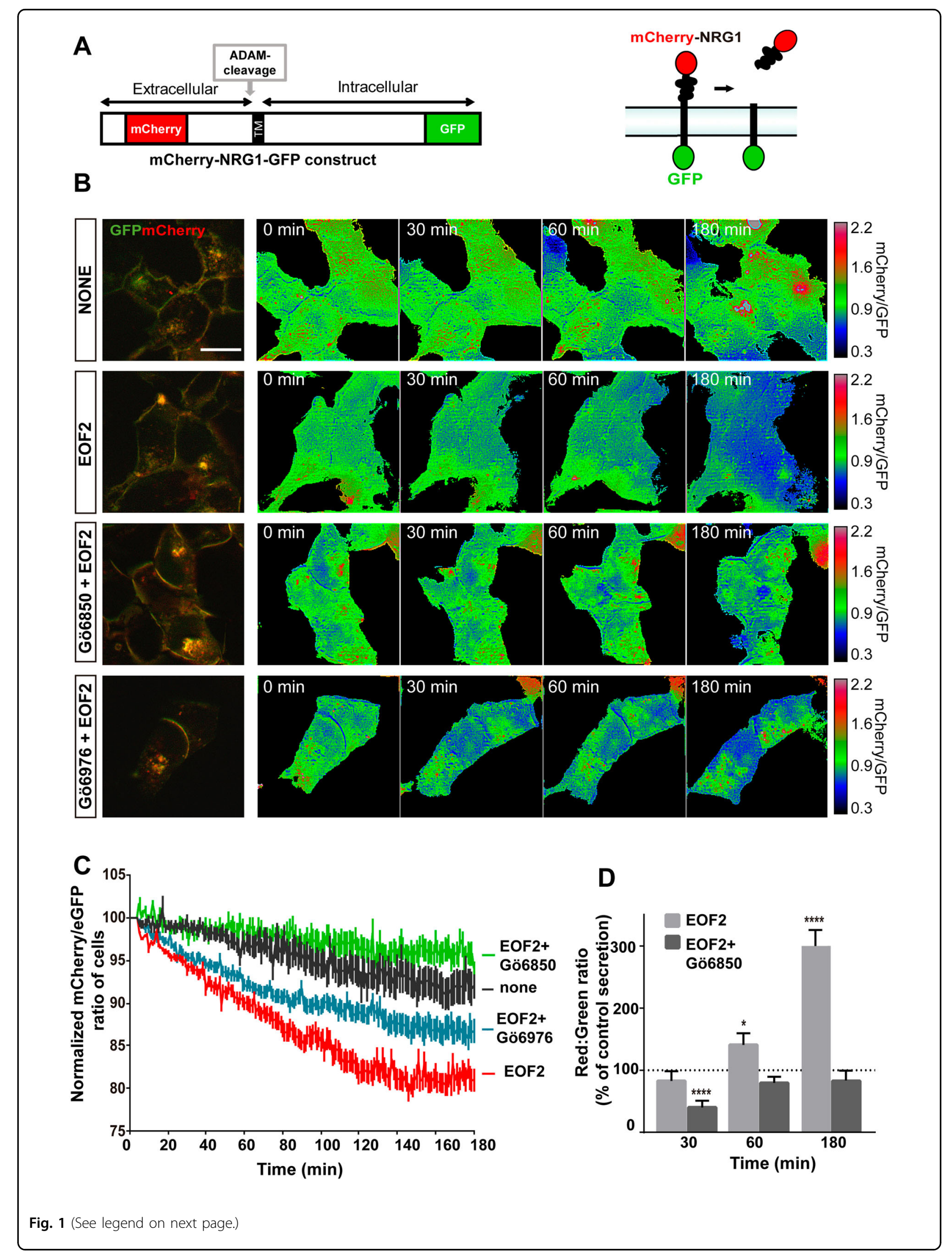


(see figure on previous page)

Fig. 1 Real-time imaging of the EOF2 induced ectodomain shedding of Cherry-NRG1-GFP. a Schematic representation of the mCherry-NRG1GFP construct and mechanism of action. $\mathbf{b}$ Images of mCherry-NRG1-GFP expressing, serum-starved HEK293T cells stimulated with diluent, EOF2 $(5 \mu \mathrm{M})$, EOF2 plus the general PKC inhibitor Gö6850 $(5 \mu \mathrm{M})$ and EOF2 plus the classical inhibitor of PKC Gö6976 (1 $\mu \mathrm{M})$ during 180 min. PKC inhibitors were added $30 \mathrm{~min}$ before EOF2. mCherry/GFP ratio images at the indicated time points are shown in the intensity modulated display-mode. The color range goes from red to blue to represent mCherry/GFP ratios. The upper and lower limits of the ratio range are shown on the right. Scale bar represent $20 \mu \mathrm{m}$. c Quantitative analysis of the microscopic images obtained from the time-lapse assays of HEK293T cells expressing Cherry-NRG1GFP. mCherry/GFP ratios were normalized to the average mCherry/GFP ratio measured before stimulation. The mean normalized mCherry/GFP ratios and SEM are shown, $n=40$. See also supplementary movie 1. d Fluorescence analysis of m-Cherry in the culture medium of HEK293T cells transfected with the construct mCherry-NRG1-GFP as a ratio of GFP fluorescence in the cellular fraction. mCherry signal in the culture medium of transfected HEK293T cells was measured in a fluorimeter as described in the Material and Methods section. Bars represent the ratio red:green in transfected cells at different time points after treatment with EOF2 or EOF2 + PKC inhibitor Gö6850. Values are given as a percentage of the red:green ratio in non-treated transfected cells (dashed line). Data are the mean values \pm S.E.M of nine independent measurements $(n=9)$. Statistical analysis: two tailed unpaired Student's $t$ test ( ${ }^{*}$ EOF2 vs control at $60 \mathrm{~min}, p=0.0456$; ${ }^{* * *}$ EOF2 + Gö6850 vs control at 30 min, $p<0.0001$; EOF2 vs control at $180 \mathrm{~min}, p<0.0001)$.

used the construct mCherry-TGF $\alpha$-eGFP (Fig. 2A) transfected into HEK293 treated with EOF2. (Fig. 2B, C). Time-lapse imaging experiments showed no reduction in the red/green ratio upon treatment with either vehicle or EOF2 (supplementary movies 5-6), whereas a time dependent reduction was observed with the pan-PKC activator PMA (supplementary movie 7). Likewise, no alteration of the red/green ratio was observed when mCherry was measured in the culture medium of mCherry-TGF $\alpha$-eGFP transfected cells at different time points (Fig. 2D).

\section{EOF2 facilitates neuronal differentiation of SVZ isolated progenitors in vitro}

To elucidate whether EOF2 played a role in neurogenesis, we tested the capacity of this compound to induce neuroblast enrichment in cultures of SVZ-isolated cells under differentiation conditions. Cells were cultured attached onto a substrate in the absence of growth factors and left for $72 \mathrm{~h}$ to differentiate in the presence and absence of EOF2. The percentage of neuroblasts ( $\beta$-IIItubulin $^{+}$) and glial cells $\left(\mathrm{GFAP}^{+}\right)$was quantified. In control cultures the percentage of $\mathrm{GFAP}^{+}$cells and $\beta$-IIItubulin $^{+}$cells were similar (Fig. 3A-D). Treatment of cultures with EOF2 increased the percentage of $\beta$-IIItubulin $^{+}$cells in almost twofold (Fig. 3A-B), whereas the percentage of $\mathrm{GFAP}^{+}$cells remained unchanged (Fig. $3 \mathrm{~A}-\mathrm{C}$ ). In addition, a small reduction in the number of nonviable cells in cultures treated with EOF2 was observed (Fig. 3D), suggesting an additional role for EOF2 in promoting survival.

\section{EOF2-induced differentiation of SVZ isolated progenitors in vitro is mediated by novel PKC}

We next study the expression patterns of the classical and novel PKC isozymes in cultures of attached SVZ isolated cells. Classical PKC $\beta$ and novel PKC , were the most abundant followed by classical $\mathrm{PKC} \alpha$ and novel $\mathrm{PKC}$. Almost undetectable levels of classical $\mathrm{PKC} \gamma$ or novel PKC $\delta$ and PKC $\eta$ were observed (Fig. 4A). Therefore, we analyzed whether blocking the expression of the most abundant novel PKC $\theta$ reverted the effect of EOF2. Attached SVZ isolated cells were cultured in the absence of growth factors and transfected with a siRNA to interfere with PKC $\theta$ expression as previously described ${ }^{26}$. Cells were left for $72 \mathrm{~h}$ in the presence and absence of EOF2 and the percentage of neuroblasts and glial cells was quantified. The elevated percentage of neuroblasts found in the presence of EOF2 was reduced to almost control levels in cultures in which PKC $\theta$ expression was inhibited by the siRNA (Fig. 4B, C). EOF2 alone or in combination with PKCO siRNA had no effect on the percentage of GFAP $^{+}$cells (Fig. 4B, D).

\section{NRG1 and ErbB4 expression in brain injuries}

In attempt to understand whether this differentiating compound could be used to promote neurogenesis in injuries, we analyzed the expression of neuregulin and its receptor ErbB4 within the perilesional area and in the adjacent SVZ in response to an injury. We performed mechanical cortical injuries in mouse brains and 14 days post injury (dpi) the expression of neuregulin and ErbB4 in the injury and in the ipsilateral SVZ was analyzed by real time qPCR. The expression of NRG1 did not change between the ipsilateral and contralateral side (Supplementary Fig. S2 B) neither within the injury or the SVZ. However, a twofold increase in ErbB4 expression was found in the ipsilateral SVZ (supplementary Fig. S2 A).

\section{Local infusion of EOF2 in brain injuries enhances the number of neuroblasts}

We next analyzed whether EOF2 infusion in brain injuries facilitated the generation of neuroblasts and neurons within the perilesional area. Mechanical injuries in the motor cortex of mouse brains were performed and EOF2 was locally infused by implanting osmotic minipumps releasing EOF2 or vehicle for 2 weeks. BrdU was injected on the day of sacrifice (Fig. 5A). Treatment 
A
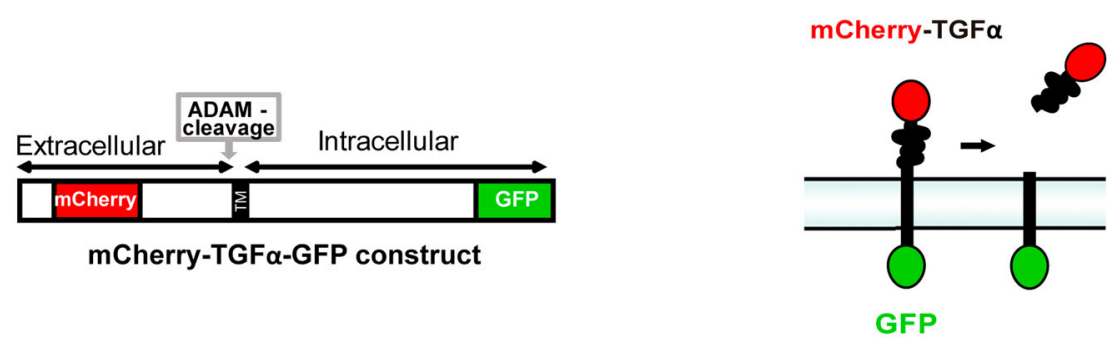

B
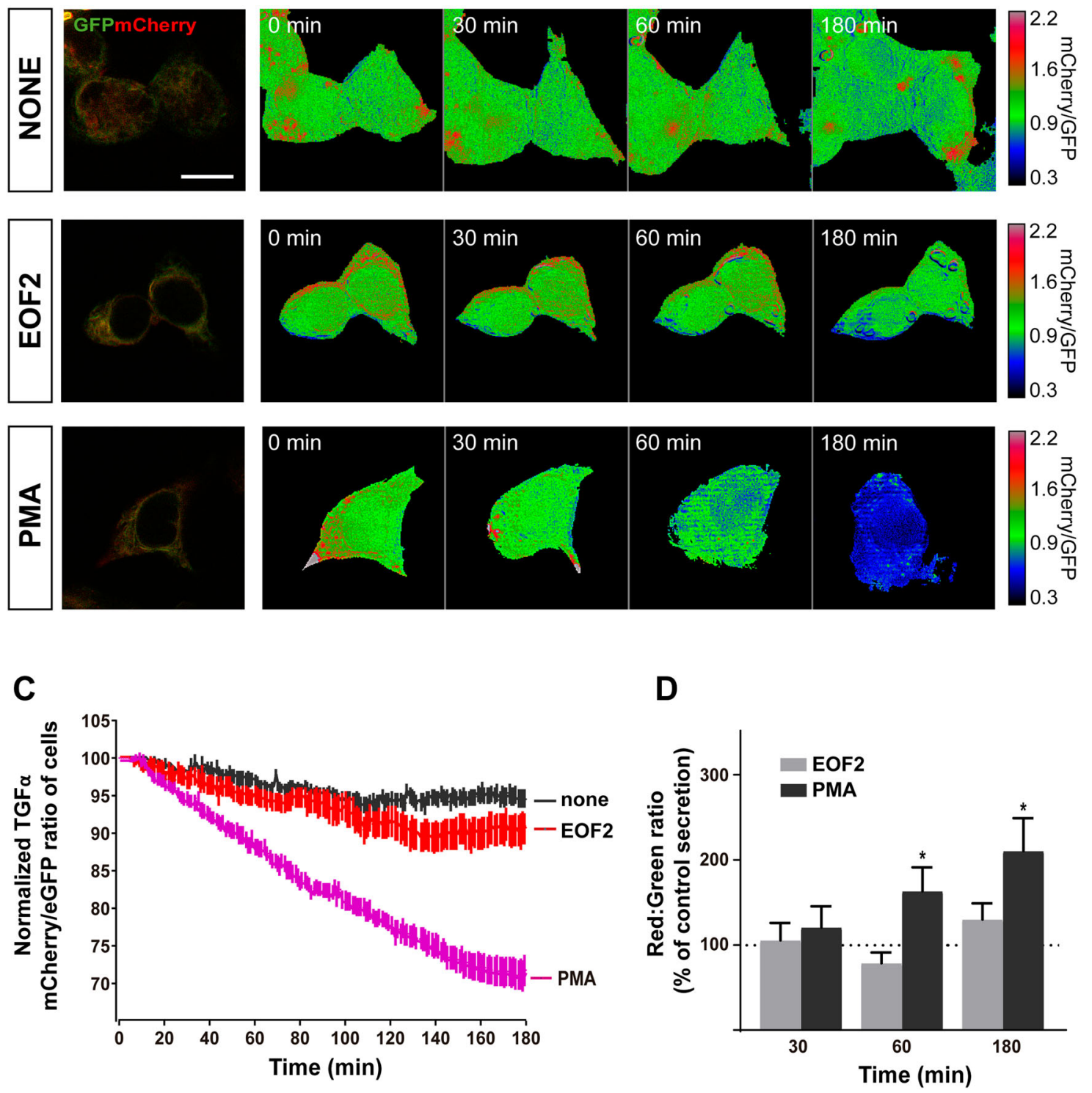

Fig. 2 (See legend on next page.)

had no effect on the extent of the injury or on the size of the perilesional area, in control and treated groups

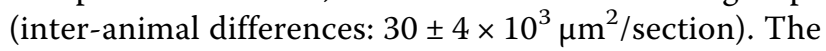
number of $\mathrm{BrdU}^{+}$cells in the perilesional area of mice treated with either vehicle or EOF2 for 14 dpi was similar between the two groups (Fig. 5B, C). Neuroblasts within the peri-lesional area of control mice were hardly visible. Interestingly, the number of neuroblasts 
(see figure on previous page)

Fig. 2 Real-time imaging of the EOF2 induced ectodomain shedding of Cherry-TGFa-GFP. a Schematic representation of the mCherry-TGFaGFP construct and mechanism of action. $\mathbf{b}$ Images of mCherry-TGFa-GFP expressing, serum-starved HEK293T cells stimulated with diluent, EOF2 $(5 \mu \mathrm{M})$ or PMA $(5 \mu \mathrm{M})$ during 180 . mCherry/GFP ratio images at the indicates time points are shown in the intensity modulated display-mode. The colors range goes from red to blue to represent mCherry/GFP ratios. The upper and lower limits of the ratio range are shown on the right. Scale bar represent $20 \mu \mathrm{m}$. c Quantitative analysis of mCherry/GFP ratios in HEK293T cells expressing Cherry-TGFa-GFP. mCherry/GFP ratios were normalized to the average mCherry/GFP ratio measured before stimulation. The mean normalized mCherry/GFP ratios and SEM are shown, $n=40$. See also supplementary movie 2 . $\mathbf{d}$ Fluorescence analysis of $\mathrm{m}$-Cherry in the culture medium of cells transfected with the fusion protein construct mCherryTGFa-GFP as a ratio of GFP fluorescence in the cellular fraction. mCherry signal in the culture medium of transfected HEK293T cells was measured in a fluorimeter as described in the Material and Methods section. Bars represent the ratio red:green in transfected cells at different time points after treatment with EOF2 or EOF2 + PKC inhibitor Gö6850 as a percentage of the red:green ratio in non-treated transfected cells (dashed line). Cells were treated with the EOF2 at the indicated times. Data are the mean values \pm S.E.M of nine independent measurements $(n=9)$. Statistical analysis: two tailed unpaired Student's $t$ test: (*PMA vs control at $60 \mathrm{~min} p=0.0403$; *PMA vs control at $180 \mathrm{~min} p=0.0112$ ).

increased dramatically in mice treated with EOF2 (Fig. 5B, D-F).

\section{Local infusion of EOF2 in brain injuries reduces the number of glial cells}

We next analyzed the number of $\mathrm{BrdU}^{+}$cells that expressed the glial marker GFAP in both control and EOF2 treated mice, as well as the area occupied by glial cells as a measure of gliosis and glial scar. Noticeable, the GFAP burden was significantly reduced in injuries treated with EOF2 compared with controls (Fig. 6A-D). Accordingly, the percentage of $\mathrm{BrdU}^{+}$cells that colocalized with the glial cell marker GFAP was also reduced in treated injuries (Fig. 6A-C). This suggested a role for EOF2 in reducing gliosis around the injured area (Fig. 6A, B). No effect of the treatment was observed on the number of nestin ${ }^{+}$undifferentiated progenitors or in its proliferation rate (Fig. 6B, E, F).

\section{Local treatment of injuries with EOF2 did not affect neurogenesis in the SVZ or DG}

It was then tested whether local treatment with EOF2 affected neurogenesis in the SVZ and DG. We analyzed $\mathrm{BrdU}^{+}, \mathrm{BrdU}^{+} / \mathrm{DCX}^{+} \mathrm{BrdU}^{+} / \mathrm{GFAP}^{+}$, and $\mathrm{BrdU}^{+} /$ nestin $^{+}$cells in both the SVZ and DG. As expected, in response to the injury, the number of $\mathrm{BrdU}^{+}$cells was significantly increased in the ipsilateral SVZ (supplementary fig. S3 A-F) and DG (supplementary fig. S4 A-F) of control and EOF2-treated mice compared with the contralateral areas, indicating the presence of a proliferative response to the injury. However, no effect of EOF2 was observed on the proliferation of progenitor cells and their progeny in the two neurogenic niches (supplementary figs. S3 and S4 A-F).

\section{Intranasal administration of EOF2 facilitates neuroblast migration towards the perilesional area}

In order to test whether neuroblast found in EOF2 treated mice had migrated from the adjacent SVZ, we had to find a method of administration that allowed EOF2 to reach neurogenic regions. In addition, used a BrdU labeling approach that allowed BrdU to incorporate in proliferating cells of the SVZ before performing the injury. Thus, healthy mice were injected with BrdU for three consecutive days and left for another 3 days for the BrdU to be cleared. By using this strategy, only proliferating cells in neurogenic niches were labeled with BrdU. Then, mice were injured and treated for 14 days with daily intranasal administrations of EOF2 or vehicle (Fig. 7A). Mice were sacrificed $14 \mathrm{dpi}$ and the colocalization of BrdU with DCX, and GFAP was quantified. Interestingly, $\mathrm{BrdU}^{+}$cells were only detected within the perilesional area of mice treated with EOF2 (Fig. 7B, C). Also, we were able to find a significant amount of $\mathrm{DCX}^{+}$ neuroblasts that were not present within the perilesional area of control mice (Fig. 7B, D). BrdU ${ }^{+}$cells were GFAP ${ }^{+}$ or $\mathrm{DCX}^{+}$(Fig. 7B, E-G). Despite the presence of $\mathrm{BrdU}^{+} /$ $\mathrm{GFAP}^{+}$cells in treated mice, no effect on the GFAP burden was observed in treated mice compared with control (Fig. 7H). The analysis of BrdU ${ }^{+}$cells in the SVZ shows a large reduction in the number of $\mathrm{BrdU}^{+}$cells in the ipsilateral SVZ of both control and EOF2 treated mice in comparison with their contralateral SVZ (supplementary fig. S5). Moreover, EOF2 treated mice showed a smaller number of $\mathrm{BrdU}^{+}$cells in the SVZ than their control counterparts (supplementary fig. S5). In addition, we have observed that neuroblasts migrated from the SVZ of EOF2 treated mice towards the injury through organized migratory pathways that described a track from the SVZ towards the injury through the corpus callosum reaching the lesioned area (supplementary fig. S6). This was not observed in control mice.

In addition, only EOF2-treated mice showed mature $\mathrm{NeuN}^{+}$neurons that had incorporated BrdU within the perilesional area (Fig. 8A, B-D). Accordingly, a larger number of mature neurons were quantified within the perilesional area of EOF2-treated mice 14 dpi (Fig. 8B, C). We next use a longer treatment (28 days) that allowed 
A
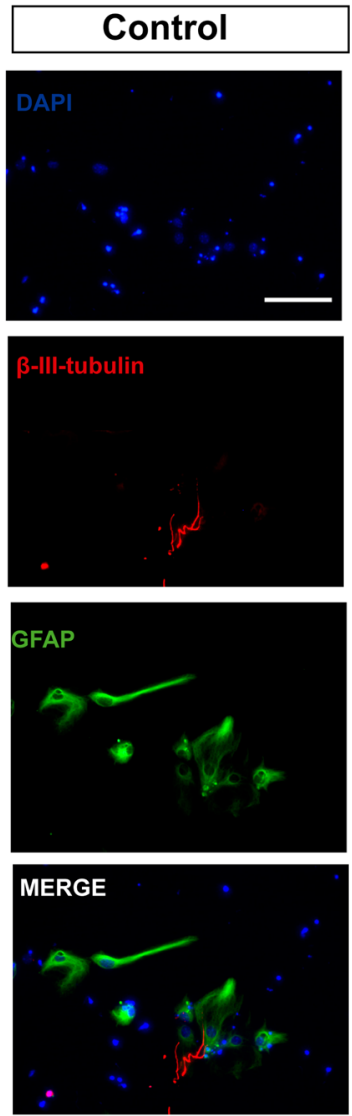
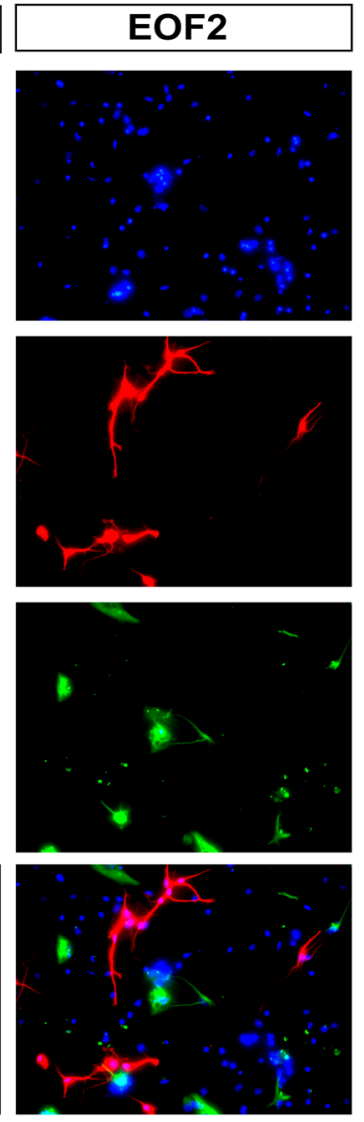

B

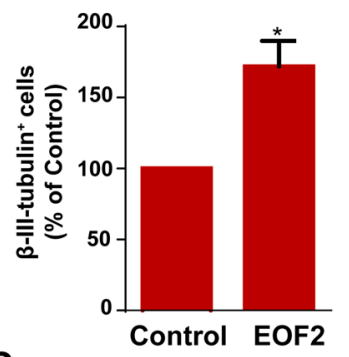

C

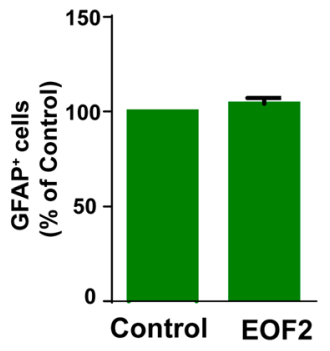

D

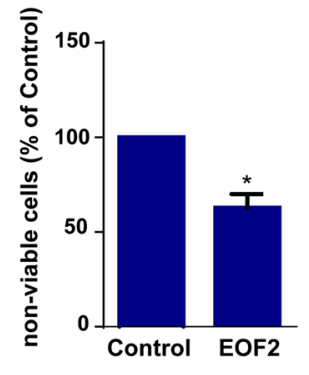

Fig. 3 EOF treatment promotes differentiation of NPC to neurons. a Representative fluorescence microphotographs of SVZ-derived cultured NPC that had been treated with either diluent (control) or EOF2 $(5 \mu \mathrm{M})$. Cells were grown without growth factors and allowed to differentiate for $72 \mathrm{~h}$ after treatment and then fixed. $\beta$-ll-tubulin marker was used for neuronal immunodetection (red) and glial cells were identified by the immunodetection of GFAP (green). Total nuclei were counterstained with DAPI (blue). Scale bar $=50 \mu \mathrm{m}$. $\mathbf{b}$ Graph represents the percentage of total cells (detected by DAPI nuclear staining) that were positive for $\beta$-III-tubulin expression expressed as percentage of control. Data are the means \pm S.E.M.; $n=9$ independent values $(n=9)$. Statistical analysis: ${ }^{*} p=0.0025$ in unpaired two tailed Student's $t$ test comparing with the control group. $\mathbf{c}$ Graph represents the percentage of total cells (detected by DAPI nuclear staining) that were positive for GFAP expression expressed as percentage of control. Data are the means \pm S.E.M. of nine independent values $(n=9)$. $\mathbf{d}$ Graph represents the percentage of nonviable cells after treatment as a percentage of total cells (detected by DAPI nuclear staining) expressed as a percentage of control. Viability was measured by trypan blue exclusion as described in the methods section. Results show a statistically significant increase in the percentage of $\beta$-lll-tubulin ${ }^{+}$cells whereas no change of $\mathrm{GFAP}^{+}$cells in the presence of EOF2. Data are the means \pm S.E.M. of nine independent values $(n=9)$. Statistical analysis: ${ }^{*} p=0.0004$ in unpaired two tailed Student's $t$ test comparing with the control group.

neuroblasts to further differentiate maintaining the BrdU labeling protocol. We analyzed the number of $\mathrm{BrdU}^{+}$cells within the perilesional area after a 28-day treatment and assessed their capacity to differentiate into mature neurons of different phenotypes. We found that the number of $\mathrm{BrdU}^{+}$cells $28 \mathrm{dpi}$ was reduced by $50 \%$ (supplementary fig. S7 A-C) compared with 14 pdi. Almost $35 \%$ of the $\mathrm{BrdU}^{+}$cells co-localized with Choline acetyl transferase $\left(\mathrm{ChAT}^{+}\right)$(supplementary fig. S7 A, D) and around 5\% colocalized with parvalbumin (supplementary fig. S7 B-D) indicating that some of the new neurons had differentiated into cholinergic and GABAergic neurons.

\section{Discussion}

We have studied in here the neurogenic effect of a small molecule, the diterpene with lathyrane skeleton EOF2. Unlike other lathyranes, EOF2 does not promote proliferation of $\mathrm{NPC}^{22}$. However, we have demonstrated that it specifically facilitates novel PKC-mediated NRG1 release, without affecting TGF $\alpha$ release. This molecule promotes neuronal differentiation of NPC in vitro in a novel PKC dependent manner. Moreover, EOF2 in vivo enables migration of neuroblasts from neurogenic areas towards an injury leading to neuroblast and neuron enrichment in damaged brain regions. 


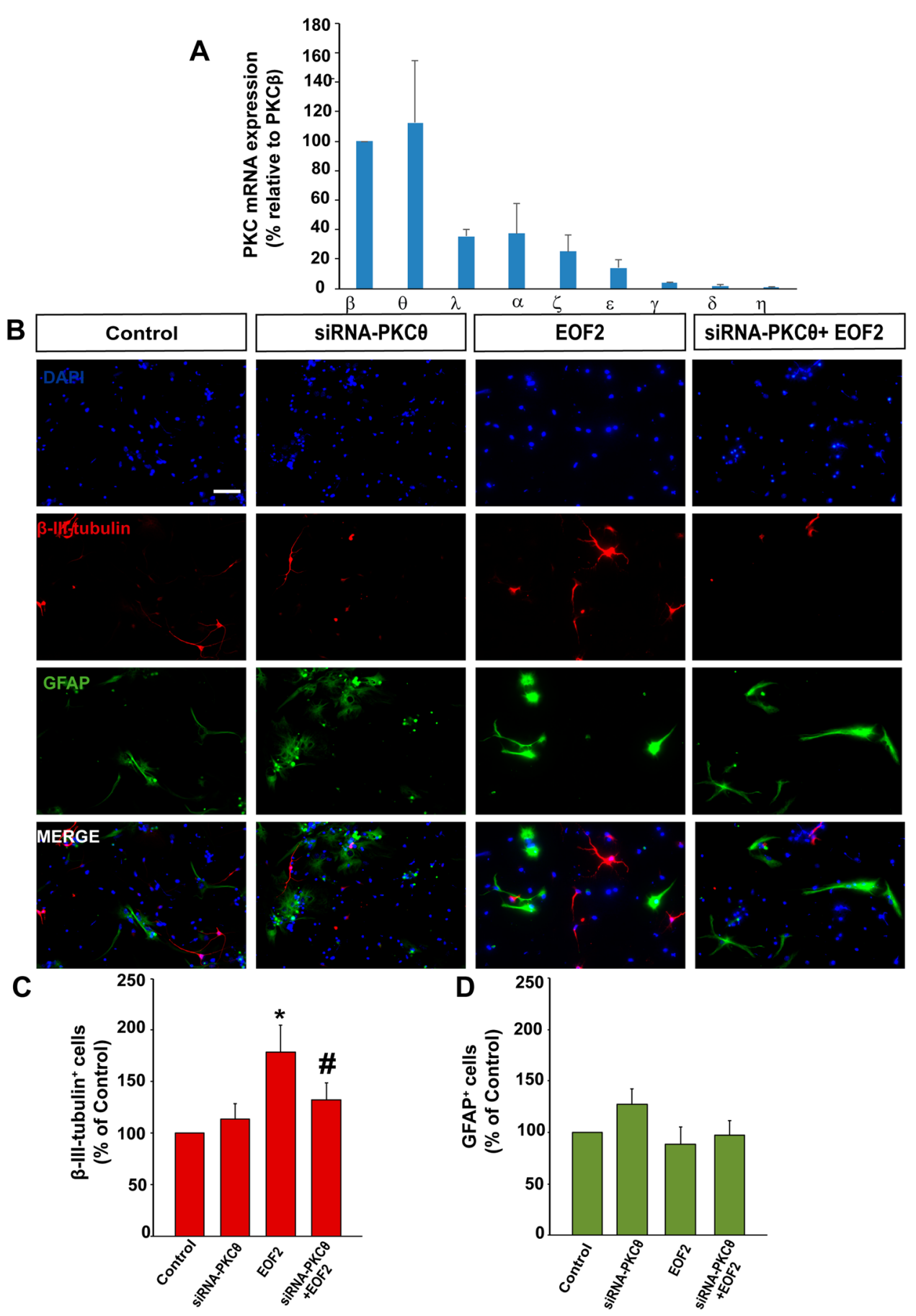

Fig. 4 EOF2 induce neuronal differentiation via PKC $\theta$ activation without affecting glia formation in NPC cultures. a Relative expression of mRNA of the different PKC isozymes under differentiation conditions. mRNA quantification was performed by reverse transcription and real time qPCR and using the $\triangle \mathrm{ct}$ method. The mRNA for PKC were measured and normalized to the levels of $18 \mathrm{~S}$ rRNA. Data are means \pm S.E.M. of five independent measurements. b Representative fluorescence microphotographs of neurosphere-derived adhered cells transfected with PKC $\theta$ siRNA, a combination of PKCO siRNA and EOF2 or either mock (control). Neuronal cells were identified by the immunocytochemical detection of $\beta$-III-tubulin (red); glial cells are identified by the immunocytochemical detection of GFAP (green) and total nuclei were counterstained with DAPI (blue). Scale bar $=50 \mu \mathrm{m}$. c Graph represents the percentage of total cells (detected by DAPI nuclear staining) that were positive for $\beta$-III-tubulin expression after treatments expressed as the percentage of control. Data are the means \pm S.E.M. of nine independent measurements $(n=9)$. Statistical analysis: two tailed unpaired Student's $t$ test of each condition compared with control ${ }^{*} p=0.003$ and \#p $\left.=0.029\right)$. $\mathbf{d}$ Graph represents the percentage of total cells (detected by DAPI nuclear staining) that were positive for GFAP expression after treatment expressed as the percentage of the control. Data are the means \pm S.E.M. of nine independent measurements $(n=9)$. 


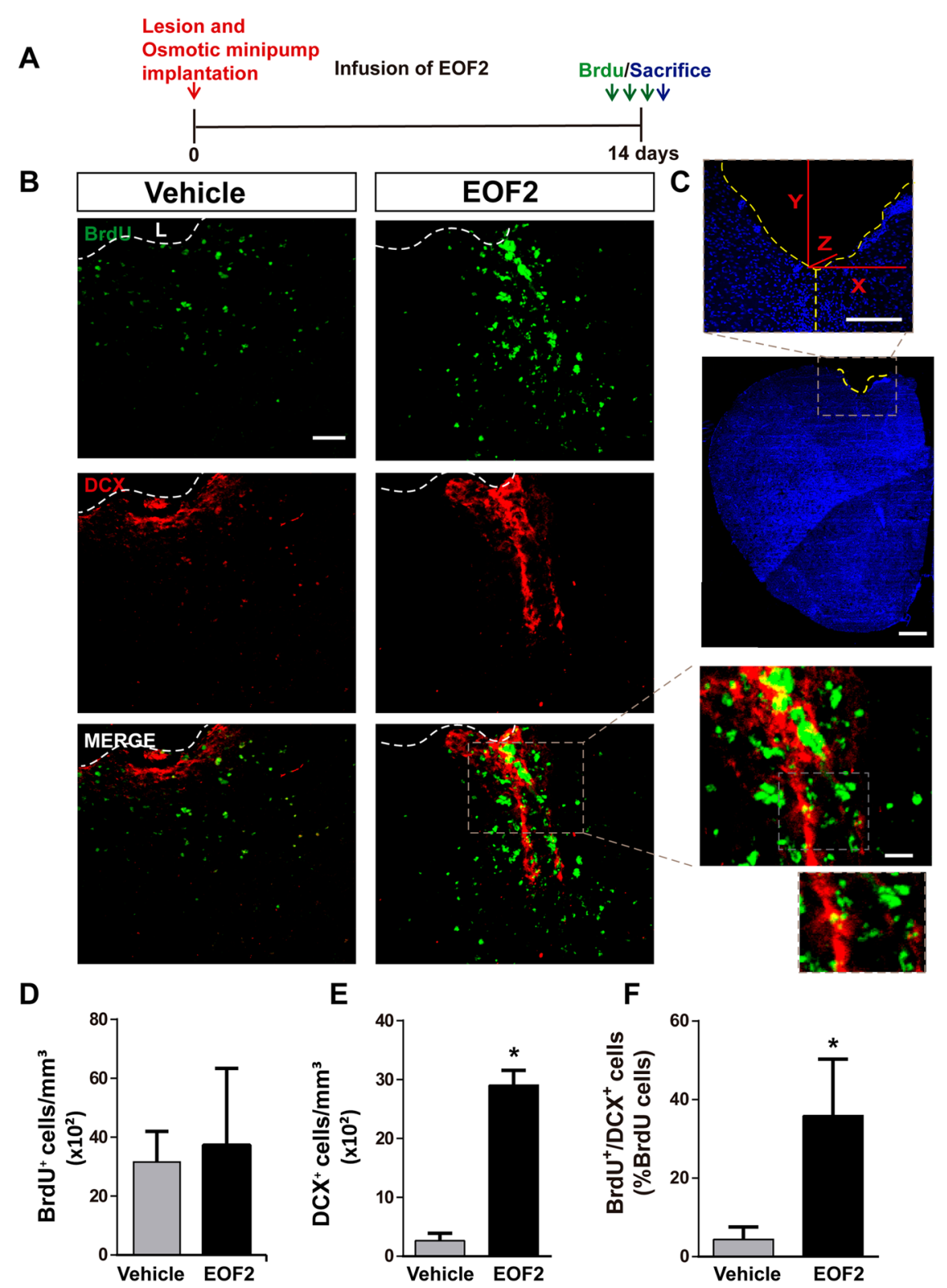

Fig. 5 Local administration of EOF2 induces neuronal differentiation in the injured cortex. a Scheme of experimental procedures. Mechanical cortical lesions were unilaterally performed in the primary motor cortex of adult mice, and osmotic minipumps were implanted to locally deliver vehicle or EOF2 $(5 \mu \mathrm{M})$ for 14 days. All mice were intraperitoneally-injected with BrdU on the last day of treatment as described in methods. b Representative confocal microphotographs of the area surrounding cortical lesion in mice brain showing immnunodetection for BrdU (upper panels) Doublecortin (DCX; medium panels) and the merged signals (lower panels). Scale bar represent $50 \mu \mathrm{m}$ in low magnification images and $20 \mathrm{\mu m}$ in high magnification images. The dotted line indicates the limit of the lesion (L). $\mathbf{c}$ Microphotograph showing details about the injury and the lesion area. In each section, the positive cells labeled with the different markers were quantified in the peri-lesional area that corresponds to $200 \mu \mathrm{m}-$ wide band of tissue surrounding the lesion border. Scale bar represent $1 \mathrm{~mm}$ in low magnification images and $200 \mu \mathrm{m}$ in high magnification images. d Graph shows the number of proliferating cells labeled with BrdU per $\mathrm{mm}^{3}$ in the peri-lesional area of the indicated animal groups. Data shown are the mean \pm S.E.M.; $n=6$ animals per group. e Quantification of $\mathrm{DCX}^{+}$(doublecortin) cells per $\mathrm{mm}^{3}$ in the peri-lesional area of the indicated animal groups. Data shown are the mean \pm S.E.M.; $n=6$ animals per group. Statistical analysis: ${ }^{*} p<0.0001$ in two tailed unpaired Student's $t$ test comparing EOF2 with the control. $\mathbf{f}$ Graph shows the percentage of $\mathrm{BrdU}^{+}$cells that co-express the neuronal marker DCX in the peri-lesional area of the indicated animal groups. Data shown are the mean \pm S.E.M.; $n=6$ animals per group. Statistical analysis: ${ }^{*} p=0.0320$ in two tailed unpaired Student's $t$ test comparing EOF2 with the control. 


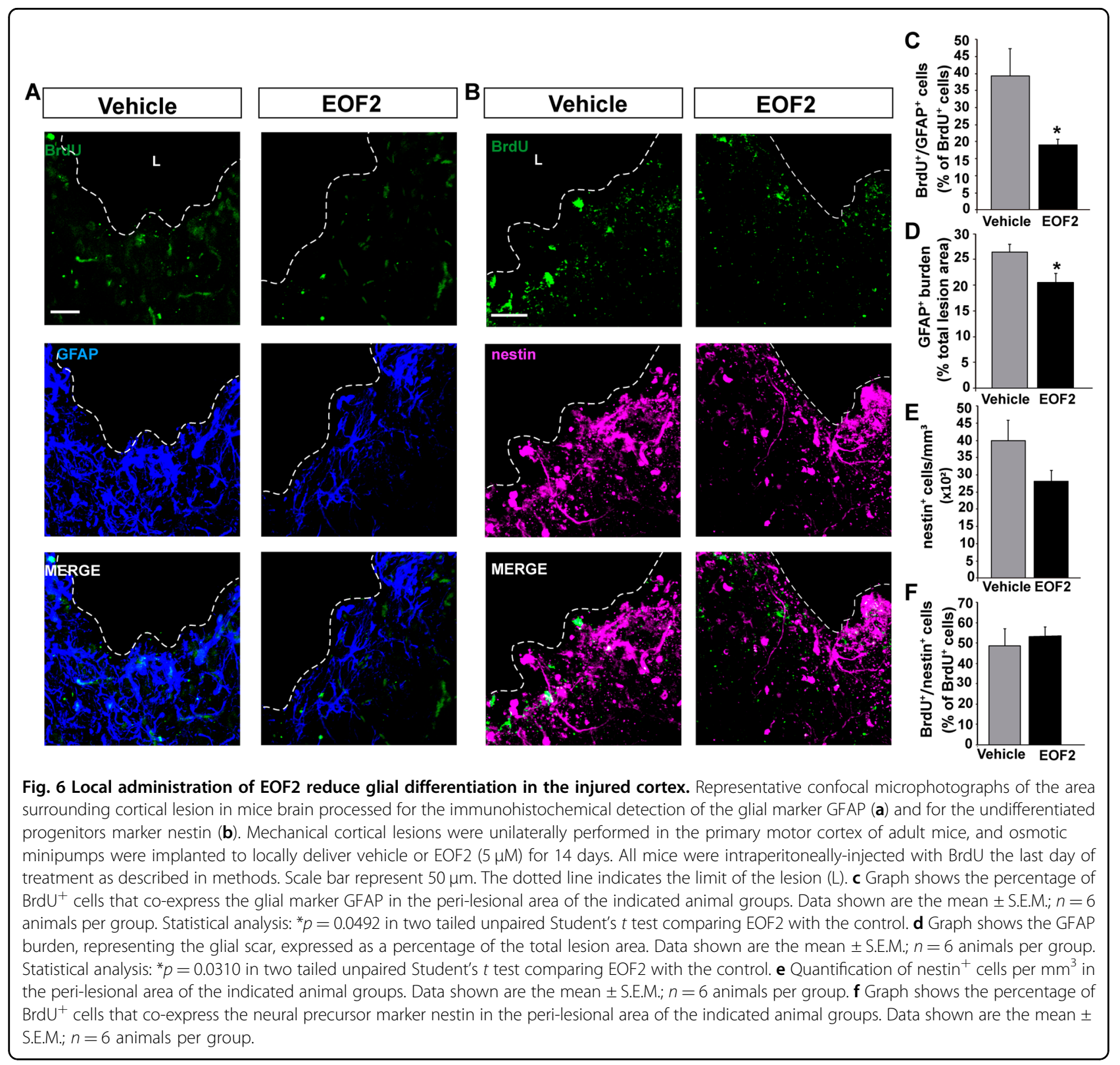

Targeting neuregulin release to promote neurogenesis in injuries

Previous reports have demonstrated the role of individual PKC isozymes as mediators of different steps in the neurogenic process ${ }^{27,28}$, some of them highlighting the role of non-tumor promoting diterpenes as PKC activators that promote neurogenesis in neurogenic niches ${ }^{22,29}$. However, neurogenic signaling molecules behave differently depending on the niche. This is the case of TGF $\alpha$, a growth factor that promotes neurogenesis in neurogenic niches $^{30}$ while inducing gliogenesis and impairing neuroblast migration in injuries ${ }^{7,11}$. Thus, a key step to facilitate neurogenesis in injuries is the introduction of neurogenic cues to counteract the gliogenic ones generated by the injury. In this work, we have focused on neuregulins, which are expressed in neuroblasts, and activate TrkA receptors on neighbor cell types. The pathways associated to these receptors promote neuroblast survival ${ }^{31}$. Also, neuregulin-mediated activation of ErbB4 receptors facilitates NSC proliferation and neuroblast migration ${ }^{20,21}$. We searched for a compound able to deliver neuregulins to the injured brain tissue. We chose to study the effect of EOF2 because of its lack of effect on stimulating NPC proliferation in a classical PKC dependent manner ${ }^{22}$ and its similarity to other classical PKC activating compounds (supplementary fig. 8). According to the report of Newton $2018^{32}$, the affinity of novel PKC isozymes for DAG and therefore for this type of 


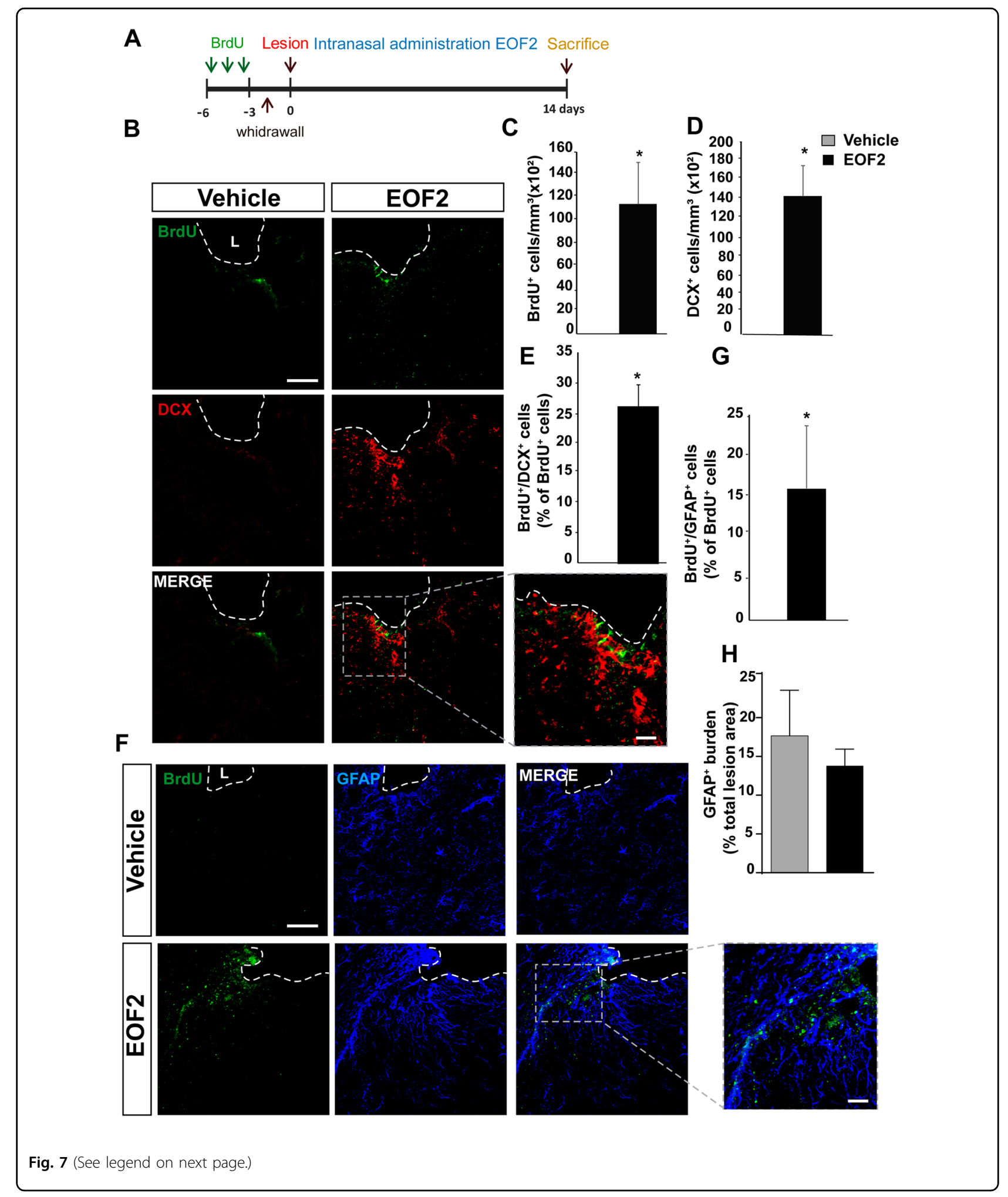

compounds is higher than that of classical PKC isozymes suggesting the possibility that EOF2 was a novel PKC activating compound with no capacity to activate the classical.
PKC isozymes participate in the release of ligands that activate ErbB receptors ${ }^{25}$. These ligands are synthesized and transported to the plasma membrane as transmembrane pro-ligands. The active soluble ligand is proteolyzed 
(see figure on previous page)

Fig. 7 Intranasal administration of EOF2 induce neuroblast migration from the neurogenic regions to the damage area. a Scheme of BrdU administration. Experimental procedure followed to label proliferating neural precursors with BrdU exclusively in neurogenic niches and not in the injured area: mice received BrdU injections on days 6, 5 and 4 before performing the cortical injury; then we waited three more days to allow for complete withdrawal of BrdU from the animal organism. Lesion was performed on day 0 . Intranasal EOF2 $(5 \mu M)$ or only vehicle was administered for 14 days. b Representative confocal microscopy images of the injured cortex of adult mice after bearing cortical lesions and the intranasal administration of EOF2 or only vehicle processed for the immunohistochemical detection of the proliferation marker BrdU and the neuroblast marker doublecortin (DCX). The dotted line indicates the limit of the lesion $(L)$ and the scale bar represent $100 \mu \mathrm{m}$ in the low magnification pictures and $50 \mu \mathrm{m}$ in the high magnification picture. c Graph shows the number of proliferating cells marked with BrdU per $\mathrm{mm}^{3}$ in the peri-lesional area of the indicated animal groups. Data shown are the mean \pm S.E.M.; $n=6$ animals per group. Statistical analysis: ${ }^{*} p=0.0377$ in two tailed unpaired Student's $t$ test comparing EOF2 with the control. d Quantification of DCX+ cells $/ \mathrm{mm}^{3}$ in the peri-lesional area of the indicated animal groups. Data shown are the mean \pm S.E.M.; $n=6$ animals per group. Statistical analysis: ${ }^{*} p=0.0012$ in two tailed unpaired Student's $t$ test comparing EOF2 with the control. e Percentage of BrdU ${ }^{+}$cells that co-expressed DCX in the peri-lesional area. Data shown are the mean \pm S.E.M.; $n=6$ animals per group. Statistical analysis: ${ }^{*} p=0.0100$ in two tailed unpaired Student's $t$ test comparing EOF2 with the control. $\mathbf{f}$ Representative confocal microscopy images of the injured cortex of adult mice in the previously indicated groups processed for the immunohistochemical detection of the proliferation marker BrdU and the glial marker GFAP. The dotted line indicates the limit of the lesion $(\mathrm{L})$ and the scale bar represent $100 \mu \mathrm{m}$ in the low magnification pictures and $50 \mu \mathrm{m}$ in the high magnification picture. $\mathbf{g}$ Percentage of $\mathrm{BrdU}^{+}$cells that co-expressed GFAP in the peri-lesional area. Data shown are the mean \pm S.E.M.; $n=6$ animals per group. Statistical analysis: ${ }^{*} p=0.0214$ in two tailed unpaired Student's $t$ test comparing EOF2 with the control. $\mathbf{h}$ Graph shows the GFAP burden within the peri-lesional area, expressed as a percentage of the total lesion area. No statistically significant differences were found. Data shown are the mean \pm S.E.M.; $n=6$ animals per group. Statistical analysis: $p=0.4464$ in two tailed unpaired Student's $t$ test comparing EOF2 with the control.

from the pro-ligand and released to the extracellular medium in a reaction catalyzed by enzymes of the ADAM family, particularly $\mathrm{ADAM} 17^{15}$. The selectivity of ADAM17 for its substrates depends on phosphorylation reactions on the pro-ligands catalyzed PKC kinases ${ }^{18,19}$. Thus, phosphorylation of TGF $\alpha$, HB-EGF, amphiregulin and other EGFR ligands by classical PKC determines their secretion, whereas, phosphorylation of NRG1 by novel PKC isozymes facilitates its release ${ }^{18,19,25}$. We used fusion proteins in which NRG1 and TGFo were fused to two fluorescent probes. Similar fusion proteins had previously been used for this particular purpose ${ }^{25}$. As time-lapse imaging fluorescence experiments show, EOF2 induces the shedding of NRG1 in HEK293T transfected cells, in a time dependent manner. The release of neuregulin to the extracellular medium was still partially observed in the presence of a classical PKC inhibitor, which at the concentration required to inhibit $\mathrm{cPKC}$ by $80 \%$, partially inhibits novel PKC by $30-40 \%{ }^{33}$. This indicates that the inhibition of classical PKC does not impair NRG1 release and suggests a role for novel PKC isozymes in the EOF2 stimulated NRG1 release. Unfortunately, commercially available specific inhibitors of novel PKC could not be used to definitely demonstrate the role of novel PKC isozymes in EOF2-mediated NRG1 release. EOF2 did not induce TGF $\alpha$ release to the extracellular medium, indicating that EOF2 specifically facilitated NRG1 release. These results agree with previous studies showing that PKC- $\delta$ is required for NRG1 cleavage, and that PKC- $\delta$ phosphorylation of serine 286 in the neuregulin cytosolic domain is essential for induced neuregulin cleavage ${ }^{18}$. Interestingly, other reports show that classical PKC activation is required for PMA-induced cleavage of all EGFR ligands ${ }^{19}$.

\section{Novel PKC-mediated differentiation of NPC into neuroblasts}

EOF2 promoted neuronal differentiation of NPC in vitro in cultures of SVZ isolated cells. This effect correlated with a reduction in the percentage of nonviable cells, suggesting an effect of EOF2 on neuroblast survival and in neuroblasts differentiation. Considering the role of neuregulins in maintaining neuroblasts survival ${ }^{31}$, it would be possible that EOF2-mediated neuregulin release facilitated neuroblasts survival in our cultures. However, an effect on NPC differentiation towards a neuronal fate is also possible, and a combination of both effects might be the reason for the elevated number of neuroblasts. The effect of EOF2 was reverted by blocking the expression of the most abundant novel PKC isozyme found in our cultures, PKCO. This result represents another evidence that supports the role of EOF2 in novel PKC activation, and neuronal differentiation probably mediated by neuregulin release.

\section{Enabling neuronal replacement in injuries with EOF2}

Previous studies describe a response of neurogenic niches to cortical and spinal cord injuries ${ }^{34-38}$. Most of these studies show a neurogenic response of the DG and SVZ together with the absence of newly generated neuroblast within the perilesional area in control conditions. Interestingly, inhibiting ADAM17-mediated release of TGF $\alpha$ resulted in a neurogenic response within the perilesional area that produced a large number of new neurons ${ }^{8}$. In agreement with these studies, we show in here that injuries of control mice treated with vehicle showed none or a very small number of neuroblasts. The importance of our work is that we find a considerable number of newborn neurons in injuries of mice treated 
A

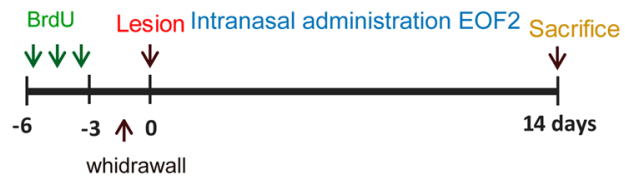

B
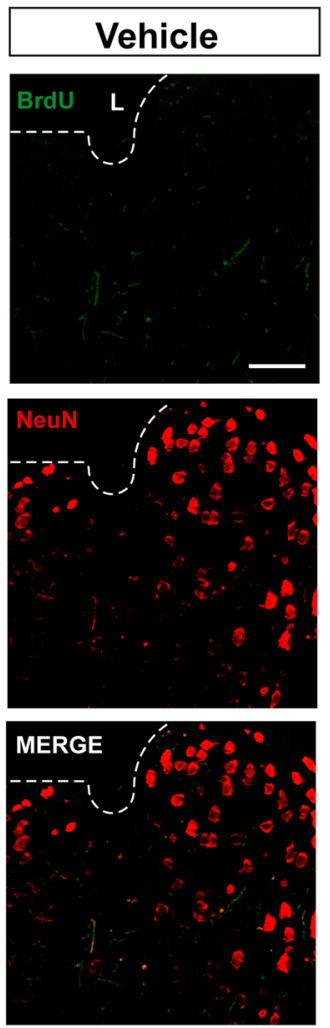
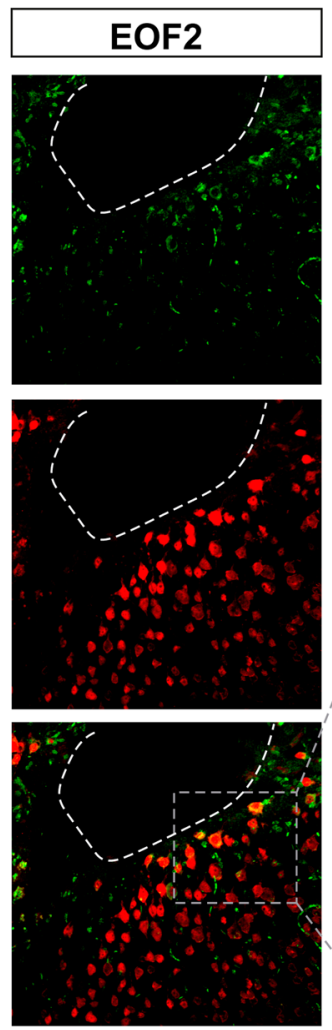

C
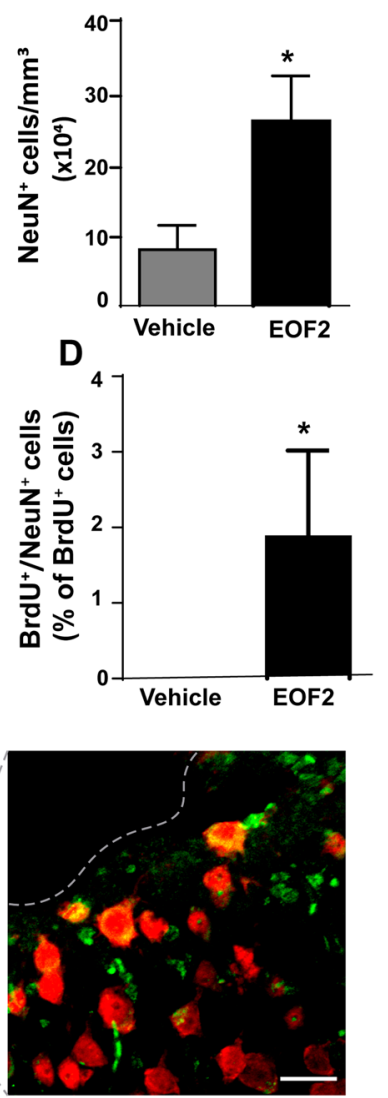

Fig. 8 Intranasal administration of EOF2 induces neuronal differentiation in the peri-lesional area after brain injury. a Scheme representing the experimental procedure followed to label proliferating neural precursors with BrdU exclusively in neurogenic niches and not in the injured area: mice received BrdU injections on days 6, 5, and 4 before performing the cortical injury; then we waited three more days to allow for complete withdrawal of BrdU from the animal organism. Lesion was performed on day 0. Intranasal administration of EOF2 ( $5 \mu \mathrm{M})$ or only vehicle was performed for 14 days. b Representative confocal microscopy images of the injured cortex of adult mice after bearing cortical lesions and the intranasal administration of EOF2 or only vehicle. The dotted line indicates the limit of the lesion $(L)$ and the scale bar represent $100 \mu \mathrm{m}$ in the low magnification pictures and $20 \mu \mathrm{m}$ in the high magnification pictures. $\mathbf{c}$ Graph shows the number of neuronal cells marked with NeuN per mm ${ }^{3}$ in the peri-lesional area of the indicated animal groups. Data shown are the mean \pm S.E.M.; $n=6$ animals per group. Statistical analysis: ${ }^{*} p=0.0339$ in two tailed unpaired Student's $t$ test comparing EOF2 with the control. d Percentage of BrdU ${ }^{+}$cells that co-expressed the neuronal marker NeuN in the peri-lesional area. Data shown are the mean \pm S.E.M.; $n=6$ animals per group. Statistical analysis: ${ }^{*} p=0.0411$ in two tailed unpaired Student's $t$ test comparing EOF2 with the control.

with EOF2. Previous results have identified neuroblasts migrating from the SVZ towards the site of injury through the corpus callosum. However, in the absence of treatments these neuroblasts never reached the injured site $^{8}$. This indicated that signaling molecules that lead neuroblasts towards the injury, need to be released-as described in Comte et al. 2011, for Galectin-3-to maintain motility from the SVZ to the olfactory bulb ${ }^{39}$. Since neuregulins play a role in neuroblast migration and survival it was reasonable to test the effect of EOF2 on migration finding an administration method that allowed the continuous administration of the compound avoiding local damage and allowing a more extensive delivery of EOF2, reaching the DG and SVZ. We used intranasal delivery, a method that has been successfully used in previous works ${ }^{40-42}$. Detection of migrating cells was possible by using a BrdU labeling strategy in which BrdU was administered to the mice 6 days prior to inducing the injury. Using this strategy, we have demonstrated that the presence of the injury induces migration of $\mathrm{BrdU}^{+}$cells from the SVZ but only in mice treated with EOF2 $\mathrm{BrdU}^{+}$ cells migrate from the SVZ reaching the injury. Moreover, in treated mice a considerable number of $\mathrm{DCX}^{+}$ neuroblasts and $\mathrm{NeuN}^{+}$neurons that co-localize with 
BrdU were found within the perilesional area $14 \mathrm{dpi}$ indicating that these neuroblasts had migrated from the SVZ. The presence of neuroblasts describing a track from the SVZ through the corpus callosum and cortex and reaching the lesioned area in treated mice supports this fact (supplementary fig. S6). An attempt of neuroblast migration was observed from the SVZ of control mice, however, no neuroblasts were found within the corpus callosum, the cortex or around the perilesional area in these mice. Neuroblasts left the SVZ and reached the corpus callosum without migrating through it towards the injury (supplementary fig. S6). After a longer 28-day treatment with EOF2 around $50 \%$ of the $\mathrm{BrdU}^{+}$cells found 14 dpi survived. At this stage, a percentage of the $\mathrm{BrdU}^{+}$cells had differentiated into mature cholinergic neurons and a few of them differentiated into parvalbumin ${ }^{+}$GABAergic neurons. The rest of $\mathrm{BrdU}^{+}$ cells, not labeled with ChAT or parvalbumin, may be neuroblasts, undifferentiated progenitors, glial cells, or they may have developed a different neuronal phenotype. In summary, all this indicates that EOF2 facilitated migration of neuroblasts and that these neuroblasts differentiated into mature neurons. Similar results have been obtained upon reduction of TGF $\alpha$ release by ADAM17 inhibition ${ }^{8}$. Therefore, it is feasible that the capacity of EOF2 to favor neuregulin release over TGF $\alpha$ is promoting neurogenesis in injuries.

\section{Conclusion}

In conclusion, we are reporting in here the discovery of EOF2 as a new non-tumorigenic specific activator of novel PKC isozymes, with the capacity to induce neurogenesis in brain injuries. We have unraveled its mechanisms of action mediated by its capacity to activate novel PKC releasing neuregulin. In addition, we have used an effective, noninvasive intranasal administration method of this compound for its effective delivery in the mouse brain promoting neuronal replacement in injuries. Our work highlights the role of novel PKC, as targets and for an effective treatment to compensate neuronal loss in damaged brain areas, being of special relevance in the development of new drugs to use in brain injury regeneration strategies.

\section{Materials and methods Reagents}

The lathyrane EOF2 3,8,12-tri-O-acetyl-7-O-(4-methoxyphenyl) acetylingol; CAS number 2230806-06-9; was isolated and purified in our laboratory, as described previously $^{43,44}$. Briefly, purification by semipreparative HPLC was performed with a Hitachi/Merck L-6270 apparatus equipped with a differential refractometer detector (RI7490). A LiChrospher ${ }^{\circledast}$ Si $60 \quad(10 \mu \mathrm{m}) \quad$ LiChroCart $^{\circledast}$ $(250 \mathrm{~mm} \times 10 \mathrm{~mm})$ column was used in isolation experiments. Silica gel (Merck) was used for column chromatography. TLC was performed on Merck Kieselgel $60 \mathrm{~F} 254,0.25 \mathrm{~mm}$ thick. Infrared spectra were recorded on a FT-IR spectrophotometer and reported as wavenumbers $\left(\mathrm{cm}^{-1}\right) .{ }^{1} \mathrm{H}$ and ${ }^{13} \mathrm{C}$ NMR measurements were obtained on a $400 \mathrm{MHz}$ spectrometer with $\mathrm{SiMe}_{4}$ as the internal reference. Chemical shifts were referenced to $\mathrm{CDCl}_{3}\left(\delta_{\mathrm{H}} 7.25, \delta \mathrm{C} 77.0\right)$, NMR assignments were made by a combination of $1 \mathrm{D}$ and $2 \mathrm{D}$ techniques. Multiplicities are described using the following abbreviations: $\mathrm{s}=$ singlet, $\mathrm{d}=$ doublet, $\mathrm{t}=$ triplet, $\mathrm{q}=$ quartet, $\mathrm{m}=$ multiplet, and $\mathrm{br}=$ broad. High-Resolution Mass Spectrometry was performed with a QTOF mass spectrometer in positive ion ESI mode.

The commercial PKC activators PMA (13-O-acetyl-12$O$-tetradecanoylphorbol, also known as TPA) and prostratin (13-O-acetyl-12-deoxyphorbol) were both purchased from Sigma-Aldrich (St. Louis, MO, USA). The general PKC inhibitor bisindolylmaleimide I (also known as GF109203X, GFX or Gö6850) and the classical PKC inhibitor Gö6976 were from Calbiochem (Millipore, Billerica, MA, USA), and were added to the cells at final concentrations of $5 \mu \mathrm{M}$ and $1 \mu \mathrm{M}$, respectively. Stock solutions of all PKC interacting compounds were prepared in DMSO and pre-diluted in culture medium before addition to cell cultures. When cultures were co-treated with PKC inhibitors and activators, PKC inhibitors were added to the cells $30 \mathrm{~min}$ before addition of PKC activators. Specific small interference RNAs (siRNAs) were from Thermo Scientific Dharmacon (Lafayette, CO, www. dharmacon.com).

Other products, unless otherwise indicated, were purchased from Sigma-Aldrich (St. Louis, MO, USA).

\section{Animal subjects}

CD1 male mice were used throughout this study. Animals were housed under controlled conditions of temperature $\left(21-23^{\circ} \mathrm{C}\right)$ and light (LD 12:12) with free access to food (AO4 standard maintenance diet, SAFE, Épinaysur-Orge, France) and water. Care and handling of animals were performed according to the Guidelines of the European Union Council (2010/63/EU), and the Spanish regulations (65/2012 and RD53/2013) for the use of laboratory animals. All studies involving animals are reported in accordance with the ARRIVE guidelines for reporting experiments involving animals ${ }^{45,46}$.

Only male mice were used in this work. The total number of animals used was: 24 2-month old adult mice and 150 7-day old pups. The number of animals used in each experiment was determined based on previous studies $^{22,29,47}$.

Adult male mice were randomized during the first week after birth by cross fostering and male were used at the age of 2 months. 
The protocol used has been authorized by the Ethics Committee of the "Consejería de Agricultura, Pesca y Desarrollo Cultural de la Junta de Andalucía”, Spain with the approval number 30/03/2016/038.

\section{SVZ cell isolation and culture}

NPC were isolated from the SVZ of 7-day postnatal (P7) mice following the same procedure described before ${ }^{48}$, and were cultured as described elsewhere ${ }^{49}$. The sacrifice of P7 mice was done by decapitation without anesthesia, and six pups were used for each independent culture. EGF $(20 \mathrm{ng} / \mathrm{ml}$, from GIBCO) and bFGF $(10 \mathrm{ng} / \mathrm{ml}$; from PeproTech, Frankfurt, Germany) were used for culture expansion, but only bFGF was present in most experimental settings, unless otherwise indicated.

\section{Cloning of human neuregulin and TGFa cDNA fused to GFP and Cherry}

Full-length cDNA encoding the membrane-bound isoform of human pro-neuregulin-1 $\beta 1$-type (NRG1, NCBI reference sequence: NP_039250.2) with mCherry cDNA inserted between nucleotides 93 and 94 of NRG1 open reading frame was cloned into pEGFP-N1 to add EGFP cDNA to the $3^{\prime}$ end. Construct was synthesized by GeneCust (Boynes, France) to generate the mCherry-NRG1GFP construct. The mCherry-TGF $\alpha$-GFP construct containing the human transforming growth factor alpha (TGFA, NCBI reference sequence: NM_003236.4), containing mCherry cDNA between nucleotides 126 and 127 of TGFA was built using the same strategy and synthesized by GeneCust (Boynes, France).

\section{HEK293T cultures and transfection}

HEK293T cells were obtained from ATCC (Manassas, VA, USA). They were cultured in DMEM at $37^{\circ} \mathrm{C}$ and $5 \%$ CO2 (Thermo Fisher Scientific, Inc., Rockford, IL, USA), supplemented with fetal bovine serum (10\%), 1× Gluta$\mathrm{MA}^{\mathrm{TM}}$-I (Thermo Fisher Scientific, Inc., Rockford, IL, USA) and penicillin/streptomycin (1\%). Cells were passaged, seeded, and allowed to attach for $24 \mathrm{~h}$. Lipofectamine 2000 (Invitrogen; Carlsbad, CA, USA) was used for transfection of these plasmids. Afterwards, medium was changed to eliminate Lipofectamine. After an overnight incubation, the cells were starved for at least $30 \mathrm{~min}$ in serum-free Fluorobrite DMEM (Thermo Fisher Scientific) containing $1 \% \mathrm{P} / \mathrm{S}, 0.25 \%$ bovine serum albumin, and $1 \times$ GlutaMAX $^{\mathrm{TM}}-\mathrm{I}$. and cells were used either in time-lapse or fluorescence experiments. Control experiments were performed to make sure that the $3.5 \mathrm{~h}$ serum starvation did not affect cell viability. The percentages of viable transfected cells in serum starved vs non-starved were $95.12 \pm 0.73$ vs $94.97 \pm 0.5$, indicating that serum deprivation for $3.5 \mathrm{~h}$ did not exert an effect on cell viability.

\section{Cell viability assays}

Cells from neurospheres were seeded at a density of 20000 cells $/ \mathrm{mL}$, and cultured in the absence or presence of specific treatments and left for $72 \mathrm{~h}$. Then, cells were detached and mixed with trypan blue [0.04\% wt/vol in phosphate-buffered saline (PBS)]. Hek293 cells were seeded at a density of 200000 cells/mL and cultured as indicated in the above section. Then cells were detached from the surface and mixed with trypan blue [0.04\% wt/ vol in phosphate-buffered saline (PBS)]. Nonviable cells (those including the trypan blue dye) and viable cells (those excluding trypan blue) were counted using a hemocytometer under an inverted microscope and were expressed as percentage of the total number of cells. Samples were coded and blinded quantifications were done in five independent experiments; each of these five independent experiments was performed in triplicate. Experiments were performed and quantified by different persons.

\section{In vitro time-lapse experiments}

Transfected HEK293T cells were plated in $35 \mathrm{~mm}$ high $\mu$-dishes (Ibidi, Munich, Germany). Cells were treated with EOF2 or inhibitors, as described in the results and figure legends. Time-lapse assays were performed with a Zeiss Axio Observer.Z1-Inverted Microscope, using a plan-apochromat 40× / 0.95 Korr M27 air objective lens. Images of transfected cells were obtained every $1 \mathrm{~min}$. Captured images were processed using ZEN lite software and the efficiency of NRG1 and TGF $\alpha$ cleavage determined by analyzing the mCherry/GFP fluorescence intensity over the entire cell areas. The mCherry/GFP ratios were calculated and normalized to the average ratio measured before stimulation with EOF2 using the Microsoft Excel software. Ratiometric images were built using ImageJ software, after background subtraction, the mCherry/GFP image was calculated dividing mCherry channel by the GFP channel. For each pixel, a pseudocolor scale is used for coding the ratio.

\section{Fluorescence analysis of mCherry-fused TGFa or neuregulin in the culture medium of HEK293T}

Transfected HEK293T cells were plated in Costar $^{\circledR} 12$ well cell culture microplate in $1 \mathrm{~mL}$ volume of culture medium. Upon treatment with the EOF2 and/or inhibitors, $50 \mu \mathrm{L}$ aliquots of culture medium were removed at 30,60 , and $180 \mathrm{~min}$ and specific mCherry fluorescence was measured using the culture medium of nontransfected cells as blank. Then, cells were washed with PBS, scratched from the surface of the plates and GFP fluorescence signal of the whole cells for each condition was determined using non-transfected cells as blank. Fluorescence of non-treated transfected cells was used as control. All fluorescence experiments were done using 
Costar $^{\circledast}$ 96-well black polystyrene plates and a BioTek ${ }^{\mathrm{TM}}$ Synergy $^{\mathrm{TM}}$ Mx Monochromator-Based MultiMode Reader. Interference fluorescence from the culture media was minimized by the optimization of the detection parameters: mCherry: excitation $587 \mathrm{~nm}$, emission $610 \mathrm{~nm}$; GFP: excitation $488 \mathrm{~nm}$, emission $510 \mathrm{~nm}$. Positive controls of TGF $\alpha$ and NRG1 shedding, after classical PKC activation were done previously using PMA and inhibitors.

\section{PKC kinase activity assay}

Neurospheres were disaggregated and 20,000 single cells were seeded per well. Treatments $(5 \mu \mathrm{M}$ EOF2 or vehicle) were added for $1 \mathrm{~h}$ prior to cell centrifugation $(200 \mathrm{~g}, 5 \mathrm{~min})$ and lysis. Inhibitors were added $30 \mathrm{~min}$ before the addition of the treatments. Protein content was measure in the lysates using the BCA method (Thermo Fisher Scientific, Rockford, IL, USA), and $1.5 \mu \mathrm{g}$ of crude protein was used per assay. The amount of PKC kinase activity was measured in each sample using the PKC Kinase Activity Assay Kit (Abcam, Cambridge, U.K.; cat. No. ab139437), following the manufacturer's instructions. Positive controls (20-60 ng of purified active PKC supplied by the kit) and blanks (diluent only) were included in each independent determination. Blanks were subtracted from measurements before comparisons were made.

\section{Cell culture adhesion, treatment, and transfection}

Neurosphere cells were centrifuged, resuspended in defined medium with growth factors, and seeded. Cell transfection with specific siRNAs was performed $18 \mathrm{~h}$ after seeding; for this, cells were changed to antibiotic-free medium and transfected using Lipofectamine 2000, following the manufacturer's instructions. Lipofectamine was removed $6 \mathrm{~h}$ later, EOF2 was added and cells were maintained for 48 additional hours before being fixed for immunocytochemistry.

\section{Immunocytochemistry}

Immunostaining for GFAP and $\beta$-III-tubulin was performed as previously described ${ }^{29}$. The primary antibodies used for this were: mouse monoclonal anti- $\beta$ III-tubulin (1:1000, Cell Signaling Technology, Boston, MA, USA) and rabbit polyclonal anti-GFAP (1:3000, Dako, Hamburg, Germany). The secondary antibodies used were: donkey anti-rabbit IgG labeled with AlexaFluor ${ }^{\oplus} 488$ (1:1000) and donkey anti-mouse IgG labeled with AlexaFluor $^{\circledR}$ 594 (1:1000), from Invitrogen (Carlsbad, CA, USA). Samples were coded before immunostaining and blinded quantifications of labeled cells were done in nine independent experiments. Experiments were performed and quantified by different individuals to avoid subjective bias during the quantification process. Original fluorescence colors have been changed in some of the pictures shown, using the Image software, to enhance visualization of markers.

\section{RNA isolation, reverse transcription and real-time quantitative PCR}

Total RNA isolation from differentiated cells, the injured and the intact cortex, reverse transcription and RT-qPCR to relatively quantify the expression of the different isozymes of PKC were performed as previously described $^{7}$. The housekeeping transcript used was $18 \mathrm{~S}$ rRNA. All PCR reaction within each experiment was run in duplicates. Amplification specificity was confirmed by melting-curve analysis of the PCR products. The relative expression of each mRNA was calculated as $2^{-\Delta \mathrm{Ct}}$, where $\Delta \mathrm{Ct}=\mathrm{Ct} \quad$ (target mRNA)-Ct (18S). No signal was detected in non-template or non-RT controls.

Primer sequences $\left(5^{\prime}-3^{\prime}\right)$ and annealing temperatures were the following: for detecting expression of mouse mRNA levels in neuroespheres we used the following primers: PKC $\alpha$, FW: TGAATCCTCAGTGGAATGAGT, RW: GGTTGCTTTCTGTCTTCTGAA, $55^{\circ} \mathrm{C}$; for PKC $\beta$, FW:CCCGAAGGAAGCGAGGGCAATGAAG, RW:AG TTCATCTGTACCCTTCCGCTCTG, $63^{\circ} \mathrm{C}$; for PKC $\gamma$, FW:TGAGAGAGTGCGGATGGGCCCC, RW:GCAGGC GTCCTGGGCTGGCACC, $65^{\circ} \mathrm{C}$; for PKC 8 , FW:GAGGC CTTGAACCAAGTGACCC, RW:CTTGCCATAGGTCC AGTTGTTG, $57^{\circ} \mathrm{C}$; for PKCe, FW:CCCATCTGAAGAC GACCGATCC, RW:CGGTTGTCAAATGACAAGGCC, $60^{\circ} \mathrm{C}$; for PKC $\theta$, FW:CCATGTCACCGTTTCTTCGA ATC, RW:TCTGCCCATTTTCTGATTCC, $56^{\circ} \mathrm{C}$; for PK C $\eta$, FW:ATGGCCACGTACCTGAGGCAGC, RW:GGA CGACGCAGGTGCACACTTGG, $65^{\circ} \mathrm{C}$; for PKC $\lambda$, FW: CGTTGGGAGCTCTGACAATC, RW:ACCTGCTTTTG CTCCATCATG, $55^{\circ} \mathrm{C}$; for PKC $\zeta$, FW:AGGAGAAGAG TACGGGTTCAGC, RW:GTGTTCATGTCAGGGTTG TCCG, $57^{\circ} \mathrm{C}$.

For mouse neuregulin and ErbB4 in injuries and SVZ we used NRG1, FW: CGCTGTTCTGGTCTCATCCG, RW: GCGGTGGAGTGGAGTGTAAG, $54^{\circ} \mathrm{C}$; for ErbB4, FW: TACCTCCTCCCATCTACACATCC, RW: CCTCT GGTATGGTGCTGGTTG, $57^{\circ} \mathrm{C}$.

The primers used for detection of human PKC isozyme mRNAs from HEK293 are described supplementary table T1.

\section{Mechanical lesions in brain cortex}

Unilateral cortical lesions were performed in the cortex of the right hemisphere of adult mice anesthetized with an intraperitoneal injection of a $100 \mathrm{mg} / \mathrm{kg}$ ketamine and $20 \mathrm{mg} / \mathrm{kg}$ xylazine cocktail. Animals were placed in a stereotaxic frame (Kopf Instruments), and a small craniotomy was performed at $+1.4 \mathrm{~mm}$ rostral and $+1.5 \mathrm{~mm}$ lateral to Bregma. A controlled mechanical lesion was performed in the underlying primary motor cortex, using 
a manually driven drill $(0.7 \mathrm{~mm}$ diameter $)$ that was allowed to penetrate $1 \mathrm{~mm}$ below the bone surface. These injuries reached the corpus callosum without damaging it.

\section{Studies describing neurogenic responses in mechanical lesions}

Mice were injured using the procedure mentioned above, and were sacrificed at 14 days post-injury (dpi). Mice were given three intraperitoneal injections of BrdU ( $70 \mathrm{mg} / \mathrm{kg}$ each) separated by $3 \mathrm{~h}$ intervals on the day of sacrifice. Mice were sacrificed by brain perfusion, and brains were processed for post-mortem studies as described below. In the same surgical acts in which cortical lesions were performed, Alzet osmotic minipumps (Charles Rivers Barcelona, Spain) were implanted subcutaneously in 12 animals and connected to infusion cannulas (brain kit II, Alzet) whose tips were placed $0.5 \mathrm{~mm}$ deep into the lesion, allowing a continuous delivery of a $5 \mu \mathrm{M}$ solution of EOF2 in PBS (containing $0.4 \%$ DMSO) or vehicle (six mice received EOF2 and six mice received vehicle). Treatments lasted for 14 days.

\section{Studies describing migration towards mechanical lesions}

In a different set of experiments, aimed to study the migration of progenitors from neurogenic regions towards the injured area, mice received BrdU injections 6, 5 , and 4 days before the injury was performed. This paradigm allowed for substantial clearance of BrdU and, therefore, it allowed BrdU labeling of NPC in the SVZ but not locally in the injured tissue. These mice (vehicle; $n=6$ and EOF2-treated; $n=6$ ) were sacrificed on day 14 after injury. Starting the day of injury, animals were treated with daily intranasal administrations of a $5 \mu \mathrm{M}$ solution of EOF2 or vehicle, until they were sacrificed $14 \mathrm{dpi}$ (Figs. 7, 8).

\section{Intranasal administration of EOF2}

EOF2, was delivered intranasally as previously described $^{40-42}$. Treatments were administered manually while the animal was placed in a standing position with an extended neck as previously described ${ }^{42} .18 \mu \mathrm{L}$ of each solution $5 \mu \mathrm{M}$ EOF2, or vehicle was delivered over both nasal cavities alternating $3 \mu \mathrm{L} /$ each using a micropipette. Mouse was maintained in such position for ten additional seconds to ensure all fluid was inhaled.

In all experiments, mice were coded, treatment (vehicle or EOF2) was assigned randomly to code numbers and applied. In addition, blind quantifications were performed to avoid subjective biases.

\section{Immunohistochemistry}

Brain processing and immunohistochemical detection of the proliferation marker BrdU, the astrocyte and neural stem cell marker GFAP, the neural progenitor cell marker nestin, the early neuronal differentiation marker doublecortin (DCX), and the mature neuron maker NeuN were performed as previously described ${ }^{49}$.

Primary antibodies used were mouse monoclonal anti-BrdU (1:100) from Dako (Hamburg, Germany) or rat monoclonal anti-BrdU (1:100) from Abcam (Cambridge, UK), mouse polyclonal anti-GFAP both from Cell Signaling (Beverly, MA, USA), goat polyclonal antiDCX (1:500), goat polyclonal anti-nestin (1:500), and mouse monoclonal anti-NeuN (1:100) all of them from Abcam (Cambridge, UK); goat anti-ChAT, polyclonal, 1:100, Merk Millipore (Billerica, MA, USA) mouse antiparvalbumin, monoclonal, 1:100, Merk Millipore (Billerica, Ma, USA). Secondary antibodies used were Alexa Fluor 488 donkey anti-mouse, Alexa Fluor 594 donkey anti-mouse, Alexa Fluor 405 goat anti-mouse, Alexa Fluor 594 donkey anti-rat, Alexa Fluor 488 donkey antirabbit, Alexa Fluor 594 donkey anti-rabbit and Alexa Fluor 594 donkey anti-goat (all at 1:1000, from Life Tech).

\section{Quantification of neurogenesis in brain sections}

SVZ cells positive for BrdU, DCX, GFAP, NeuN, and nestin were estimated as previously described ${ }^{29,49}$. Positive cells were counted throughout the entire perilesional area or lateral and laterodorsal walls of the lateral ventricles (where the SVZ NPC are located) in every fifth section; 14-16 sections per brain where analyzed under fluorescence microscopy at $\times 20$ magnification. A confocal microscope (OLYMPUS FV1000) was used to take images from triple-labeled brain sections. Mice were coded depending on the treatment and quantification of cells in brain slices was done in blinded analysis.

\section{Statistical analysis}

The data and statistical analysis comply with the recommendations on experimental design and analysis in pharmacology ${ }^{50}$. Statistical analysis was performed using the computer program IBM SPSS Statistics 22. Normal distribution of the data was first analyzed using a Shapiro-Wilks test. Then, a Brown Forsythe test was performed to test the equality of variances. Afterwards, when more than one treatment group were compared, statistical analyses were performed using one-way ANOVA followed by a post-hoc Bonferroni's test unless otherwise indicated. A Student's $t$ test was used when only one treatment group was compared with the control. Differences were considered significant at values of $p<$ 0.05 . In general, sample size used in statistical analysis were $n=6$ for in vivo experiments and $n=5-9$ for in vitro experiments. Sample sizes were chosen based on a previous works related to this one $e^{8,22,29,47}$. 


\section{Acknowledgements}

We dedicate this paper to our late colleague and friend, Dr. Maribel MurilloCarretero, whose dedication and critical advice was very valuable to this work. This work was supported by the Spanish Ministerio de Ciencia, Innovación y Universidades (Grant Numbers RTI2018-099908-B-C21, and RTI2018-099908-BC21 MICINN/FEDER granted to CC and RHG respectively and BFU2016-75038R granted to MGA) and Consejería de Economía, Conocimiento, Empresas y Universidades Junta de Andalucía (grant number FEDER-ANDALUCÍA sol2018-00106647-tra). We thank Antonio Torres for his technical assistance. We thank the Servicio de experimentación y producción animal (SEPA) de la Universidad de Cádiz as well as the Servicios Centrales de apoyo a la investigación en Ciencias de la Salud (SCICS) and Servicios centrales de Ciencia y tecnología (SC-ICYT) de la Universidad de Cádiz. We appreciate the assistance provided by Juan Luis Ribas and Modesto Carballo of the Servicio de Microscopia y Biología, respectively, of the Centro de Investigación, Tecnología e Innovación de la Universidad de Sevilla (CITIUS).

\section{Author details}

'Área de Fisiología, Facultad de Medicina, Universidad de Cádiz, Cádiz, Spain. ${ }^{2}$ Instituto de Investigación e Innovación Biomédica de Cádiz (INiBICA), Cádiz, Spain. ${ }^{3}$ Departamento de Anatomía y Embriología Humanas, Facultad de Medicina, Universidad de Cádiz, Cádiz, Spain. ${ }^{4}$ Área de Nutrición, Facultad de Medicina Universidad de Cádiz, Cádiz, Spain. ${ }^{5}$ Departamento de Fisiología, Facultad de Farmacia, Universidad de Sevilla, Sevilla, Spain. ${ }^{6}$ Área de Bioquímica, Facultad de Ciencias, Universidad de Cádiz, Puerto Real, Spain. ${ }^{7}$ Departamento de Química Orgánica, Facultad de Ciencias, Universidad de Cádiz, Puerto Real, Spain

\section{Conflict of interest}

The authors declare that they have no conflict of interest.

\section{Publisher's note}

Springer Nature remains neutral with regard to jurisdictional claims in published maps and institutional affiliations.

Supplementary Information accompanies this paper at (https://doi.org/ 10.1038/s41419-020-2453-9).

Received: 2 September 2019 Revised: 2 April 2020 Accepted: 3 April 2020 Published online: 22 April 2020

\section{References}

1. Blennow, K, Hardy, J. \& Zetterberg, H. The neuropathology and neurobiology of traumatic brain injury. Neuron 76, 886-899 (2012).

2. Gage, F. H. et al. Survival and differentiation of adult neuronal progenitor cells transplanted to the adult brain. Proc. Natl Acad. Sci. USA 92, 11879-11883 (1995).

3. Doetsch, F., Garcia-Verdugo, J. M. \& Alvarez-Buylla, A. Cellular composition and three-dimensional organization of the subventricular germinal zone in the adult mammalian brain. J. Neurosci. 17, 5046-5061 (1997).

4. Jin, K. et al. Neurogenesis in dentate subgranular zone and rostral subventricular zone after focal cerebral ischemia in the rat. Proc. Natl Acad. Sci. USA 98, 4710-4715 (2001).

5. Liu, J., Solway, K., Messing, R. O. \& Sharp, F. R. Increased neurogenesis in the dentate gyrus after transient global ischemia in gerbils. J. Neurosci. 18, 7768-7778 (1998).

6. Fallon, J. et al. In vivo induction of massive proliferation, directed migration, and differentiation of neural cells in the adult mammalian brain. Proc. Nat Acad. Sci. USA 97, 14686-14691 (2000).

7. Romero-Grimaldi, C. et al. ADAM-17/tumor necrosis factor-alpha-converting enzyme inhibits neurogenesis and promotes gliogenesis from neural stem cells. Stem Cells 29, 1628-1639 (2011).

8. Geribaldi-Doldan, N. et al. Specific inhibition of ADAM17/TACE promotes neurogenesis in the injured motor cortex. Cell Death Dis. 9, 862 (2018).

9. Susarla, B. T., Villapol, S., Yi, J. H., Geller, H. M. \& Symes, A. J. Temporal patterns of cortical proliferation of glial cell populations after traumatic brain injury in mice. ASN Neuro. 6, 159-170 (2014).
10. Buffo, A. et al. Origin and progeny of reactive gliosis: a source of multipotent cells in the injured brain. Proc. Natl Acad. Sci. USA 105, 3581-3586 (2008).

11. Kim, Y., Comte, I., Szabo, G., Hockberger, P. \& Szele, F. G. Adult mouse subventricular zone stem and progenitor cells are sessile and epidermal growth factor receptor negatively regulates neuroblast migration. PLOS ONE 4, e8122 (2009).

12. Kuhn, H. G., Winkler, J., Kempermann, G., Thal, L. J. \& Gage, F. H. Epidermal growth factor and fibroblast growth factor-2 have different effects on neural progenitors in the adult rat brain. J. Neurosci. 17, 5820-5829 (1997).

13. Gonzalez-Perez, O., Romero-Rodriguez, R., Soriano-Navarro, M., Garcia-Verdugo, J. M. \& Alvarez-Buylla, A. Epidermal growth factor induces the progeny of subventricular zone type B cells to migrate and differentiate into oligodendrocytes. Stem Cells 27, 2032-2043 (2009).

14. Dominguez-Garcia, S., Castro, C. \& Geribaldi-Doldan, N. ADAM17/TACE: a key molecule in brain injury regeneration. Neural Regen. Res. 14, 1378-1379 (2019).

15. Blobel, C. P. ADAMs: key components in EGFR signalling and development. Nat. Rev. 6, 32-43 (2005).

16. Lee, D. C. et al. TACE/ADAM17 processing of EGFR ligands indicates a role as a physiological convertase. Ann. N. Y. Acad. Sci. 995, 22-38 (2003).

17. Sunnarborg, S. W. et al. Tumor necrosis factor-alpha converting enzyme (TACE) regulates epidermal growth factor receptor ligand availability. J. Biol. Chem. 277, 12838-12845 (2002).

18. Dang, M. et al. Regulated ADAM17-dependent EGF family ligand release by substrate-selecting signaling pathways. Proc. Natl Acad. Sci. USA 110, 9776-9781 (2013).

19. Dang, M. et al. Epidermal growth factor (EGF) ligand release by substratespecific a disintegrin and metalloproteases (ADAMs) involves different protein kinase C (PKC) isoenzymes depending on the stimulus. J. Biol. Chem. 286, 17704-17713 (2011)

20. Anton, E. S. et al. Receptor tyrosine kinase ErbB4 modulates neuroblast migration and placement in the adult forebrain. Nat. Neurosci. 7, 1319-1328 (2004).

21. Ghashghaei, H. T. et al. The role of neuregulin-ErbB4 interactions on the proliferation and organization of cells in the subventricular zone. Proc. Natl Acad. Sci. USA 103, 1930-1935 (2006).

22. Murillo-Carretero, M. et al. ELAC (3,12-di-O-acetyl-8-O-tigloilingol), a plantderived lathyrane diterpene, induces subventricular zone neural progenitor cell proliferation through PKCbeta activation. Br. J. Pharm. 174, 2373-2392 (2017).

23. Geribaldi-Doldan, N., Gomez-Oliva, R., Dominguez-Garcia, S., Nunez-Abades, P. \& Castro, C. Protein Kinase C: targets to regenerate brain injuries? Front. Cell Dev. Biol. 7, 39 (2019).

24. Flores-Giubi, E. et al. Lathyrane, premyrsinane, and related diterpenes from euphorbia boetica: effect on in vitro neural progenitor cell proliferation. J. Nat. Prod. 82, 2517-2528 (2019).

25. Kamezaki, A. et al. Visualization of Neuregulin 1 ectodomain shedding reveals its local processing in vitro and in vivo. Sci. Rep. 6, 28873 (2016).

26. Garcia-Bernal, F. et al. Protein Kinase C inhibition mediates neuroblast enrichment in mechanical brain injuries. Front. Cell Neurosci. 12, 462 (2018).

27. Wang, J. et al. Metformin activates an atypical PKC-CBP pathway to promote neurogenesis and enhance spatial memory formation. Cell Stem Cell 11, 23-35 (2012).

28. Fatt, M. et al. Metformin acts on two different molecular pathways to enhance adult neural precursor proliferation/self-renewal and differentiation. Stem Cell Rep. 5, 988-995 (2015).

29. Rabaneda, L. G. et al. Altered regulation of the Spryz/Dyrk1A/PP2A triad by homocysteine impairs neural progenitor cell proliferation. Biochim Biophys. Acta 1863, 3015-3026 (2016).

30. Tropepe, V., Craig, C. G., Morshead, C. M. \& van der Kooy, D. Transforming growth factor-alpha null and senescent mice show decreased neural progenitor cell proliferation in the forebrain subependyma. J. Neurosci. 17, 7850-7859 (1997)

31. Verdi, J. M. et al. A reciprocal cell-cell interaction mediated by NT-3 and neuregulins controls the early survival and development of sympathetic neuroblasts. Neuron 16, 515-527 (1996).

32. Newton, A. C. Protein kinase C: perfectly balanced. Crit. Rev. Biochem. Mol. Biol. 53, 208-230 (2018)

33. Wu-Zhang, A. X. \& Newton, A. C. Protein kinase C pharmacology: refining the toolbox. Biochem. J. 452, 195-209 (2013).

34. Cavanagh, J. B. The proliferation of astrocytes around a needle wound in the rat brain. J. Anat. 106, 471-487 (1970). 
35. Adrian, E. K. Jr. \& Walker, B. E. Incorporation of thymidine-H3 by cells in normal and injured mouse spinal cord. J. Neuropathol. Exp. Neurol. 21, 597-609 (1962).

36. Chirumamilla, S., Sun, D., Bullock, M. R. \& Colello, R. J. Traumatic brain injury induced cell proliferation in the adult mammalian central nervous system. J. Neurotrauma 19, 693-703 (2002).

37. Dash, P. K., Mach, S. A. \& Moore, A. N. Enhanced neurogenesis in the rodent hippocampus following traumatic brain injury. J. Neurosci. Res. 63, 313-319 (2001).

38. Rice, A. C. et al. Proliferation and neuronal differentiation of mitotically active cells following traumatic brain injury. Exp. Neurol. 183, 406-417 (2003)

39. Comte, I. et al. Galectin-3 maintains cell motility from the subventricular zone to the olfactory bulb. J. Cell Sci. 124, 2438-2447 (2011).

40. Thorne, R. G., Pronk, G. J., Padmanabhan, V. \& Frey, W. H. 2nd Delivery of insulin-like growth factor-I to the rat brain and spinal cord along olfactory and trigeminal pathways following intranasal administration. Neuroscience 127 481-496 (2004).

41. Francis, G. J. et al. Intranasal insulin prevents cognitive decline, cerebral atrophy and white matter changes in murine type I diabetic encephalopathy. Brain 131, 3311-3334 (2008).

42. Marks, D. R., Tucker, K., Cavallin, M. A., Mast, T. G. \& Fadool, D. A. Awake intranasal insulin delivery modifies protein complexes and alters memory, anxiety, and olfactory behaviors. J. Neurosci. 29, 6734-6751 (2009).

43. Zhao, N. D. et al. Identification of ingol and rhamnofolane diterpenoids from euphorbia resinifera and their abilities to induce lysosomal biosynthesis. J. Nat. Prod. 81, 1209-1218 (2018).
44. Avila, L. et al. Effects of diterpenes from latex of Euphorbia lactea and Euphorbia laurifolia on human immunodeficiency virus type 1 reactivation. Phytochemistry 71, 243-248 (2010).

45. McGrath, J. C., Drummond, G. B., McLachlan, E. M., Kilkenny, C. \& Wainwright, C L. Guidelines for reporting experiments involving animals: the ARRIVE guidelines. Br. J. Pharm. 160, 1573-1576 (2010).

46. Kilkenny, C. et al. Animal research: reporting in vivo experiments: the ARRIVE guidelines. Br. J. Pharm. 160, 1577-1579 (2010).

47. Carrasco, M. et al. Glycine N-methyltransferase expression in the hippocampus and its role in neurogenesis and cognitive performance. Hippocampus 24 840-852 (2014).

48. Torroglosa, A. et al. Nitric oxide decreases subventricular zone stem cell proliferation by inhibition of epidermal growth factor receptor and phosphoinositide-3-kinase/Akt pathway. Stem Cells 25, 88-97 (2007).

49. Rabaneda, L. G. et al. Homocysteine inhibits proliferation of neuronal precursors in the mouse adult brain by impairing the basic fibroblast growth factor signaling cascade and reducing extracellular regulated kinase 1/2-dependent cyclin E expression. FASEB J. 22, 3823-3835 (2008).

50. Curtis, M. J. \& Abernethy, D. R. Revision of instructions to authors for pharmacology research and perspectives: enhancing the quality and transparency of published work. Pharmacol. Res. Perspect. 3, https://doi.org/10.1002/prp2.106 (2015). 